\title{
Multi-Level Fast Multipole Algorithm for General Targets on a Half-Space Interface
}

\author{
Zhijun Liu, Jiangqi He, Yongjun Xie, ${ }^{1}$ Anders Sullivan and Lawrence Carin \\ Department of Electrical and Computer Engineering \\ Duke University \\ Durham, NC 27708-0291 \\ ${ }^{1}$ Army Research Laboratory \\ Adelphi, MD
}

\begin{abstract}
The multi-level fast multipole algorithm (MLFMA) is considered for scattering from an electrically large conducting or dielectric target resting on the interface of a dielectric half space. We focus on analysis of the half-space Green's function such that it is computed efficiently and accurately, while retaining a form that is applicable to an MLFMA analysis. Attention is also directed toward development of a simple preconditioner to accelerate convergence of the conjugategradient solver. The utility of the model is examined for several applications, including scattering from an electrically large vehicle, trees and rough dielectric interfaces in the presence of a dielectric half space background.
\end{abstract}

\section{Introduction}

There are many applications for which one is interested in scattering from electrically large targets situated in the presence of a lossy half space. In the context of natural targets encountered in radar, one is interested in scattering from a rough-soil interface, as well as from vegetation. There is also significant interest in scattering from ground-based made-made targets, such as vehicles [1]. One of the principal tools for the analysis of such scattering is the method of moments (MoM) $[2,3]$, with such recently extended via the fast multipole method (FMM) and the multi-level fast multipole algorithm (MLFMA) [1,4-8]. In such formulations one of the most complicated issues is evaluation of the half-space dyadic Green's function [1-3]. Each component of the dyadic is represented in general via a Sommerfeld integral, the direct numerical evaluation of which makes a MoM analysis

prohibitive. Moreover, the FMM and MLFMA models were developed originally in the context of the free-space Green's function [4], such not directly transferable to the half-space problem. To 
address this issue, one could evaluate the Sommerfeld integrals via the complex-image technique [1-3,9], wherein each component of the dyadic is represented in terms of a sum of free-space Green's functions, with sources in complex space [9]. This method has been employed previously in the context of the MoM [2,3]. However, it has been demonstrated that the FMM identities are in general slowly convergent for sources in complex space [1,7]. Consequently, in previous FMM and MLFMA studies [1,7] the "near" terms are evaluated via the complex-image technique, as in the MoM $[2,3]$, and the "far" FMM/MLFMA terms are evaluated via an approximation to the dyadic Green's function, using a single appropriately weighted image in real space.

While these initial FMM and MLFMA models for scattering near a half-space interface have been found to be highly accurate [1,7], the aforementioned approximation to the Green's function limits the range of model applicability. In particular, the real-image representation of the Green's function is analogous to a first-order asymptotic steepest-descent-path (SDP) evaluation, this appropriate for expansion and testing function separated by a wavelength or more [10]. This is typically appropriate for the "far" FMM/MLFMA terms, although we have found that the accuracy of this first-order approximation deteriorates as the target becomes close to the half-space interface.

In this paper we consider a higher-order approximation for efficient evaluation of the half-space Green's function, with the resulting construct still applicable to the FMM formulation. The approximation is principally based on employing higher-order asymptotic terms, and it is demonstrated that this approximation yields highly accurate results, with comparisons to a MoM analysis in which the Green's-function Sommerfeld integrals are evaluated rigorously [2,3]. The resulting MLFMA therefore employs two distinct means of treating the Sommerfeld integrals. For the "far" terms, handled using the MLFMA propagator between clusters [1,4-8], the Green's function is evaluated via the aforementioned higher-order asymptotic model. The remaining "near" interactions are evaluated as in a MoM analysis, and the Sommerfeld integrals are evaluated accurately using the complex-image technique $[2,3,9]$.

Both the complex-image technique and the higher-order asymptotic model can be viewed in terms of image sources, constituting the reflected term in the half-space Green's function. In the 
work reported here the targets of interest touch the half space interface. In this case the image sources can be very near to or even touch some of the testing functions on the target surface. We therefore demonstrate the need to perform singularity extraction [11] when evaluating the fields radiated by such image sources, for accurate computation of the associated (expansion function)-(testing function) interactions.

As discussed above, there are many applications for which one is interested in scattering from dielectric targets in the vicinity of a half space. For example, in the context of scattering from a rough dielectric surface, surface truncation is an important issue. In previous rough-surface studies, beam excitation is often employed [12], in an attempt to mitigate scattering artifacts introduced by the rough-surface truncation. In the work reported here the rough surface is placed in the presence of an infinite half space, and the surface is represented as a dielectric target composed of the same electrical properties as the background subsurface. By tapering the transition from the half space to the rough interface, and placing the target in direct contact with the dielectric half space, one realizes a smooth transition between an infinite planar surface and a rough region. We pursue this approach in the examples presented below, with the problem solved via the aforementioned MLFMA formulation. As we demonstrate, the rough surface is modeled by a thin dielectric volume, with the solution realized via a surface-integral-equation formulation. Because of the thin nature of this target, we have found that the scattering formulation is poorly conditioned, leading to slow convergence of the conjugate-gradient (CG) solver.

It is therefore desirable to develop a simple preconditioner to enhance the CG convergence rate, this of interest for scattering from general electrically large targets, the rough surface representing a special case. Therefore, we have implemented a simple preconditioner that was first introduced in the mathematics literature [13,14], it particularly useful for dielectric targets. As discussed in detail below, this preconditioner is simple to implement, and in many cases we have found it to increase the CG convergence rate by a factor of two.

The remainder of the text is organized as follows. In Sec. II we discuss the higher-order asymptotic model used to evaluate the half-space Green's function, in the context of the far MLFMA 
interactions. We also underscore the need to carefully treat the Green's-function singularities for the image-like terms, when the target is near or touches the interface. The simple preconditioner is discussed in Sec. III. Several example results are presented in Sec. IV, including scattering from canonical targets (with comparison to a reference MoM solution) as well as from tree-like targets and a rough dielectric interface. The paper is summarized and conclusions are drawn in Sec. V.

\section{Half-Space Green's Function Evaluation}

\section{A. Asymptotic form of spectral reflection coefficient}

In an MoM analysis one must compute order $N^{2}$ interactions between $N$ basis functions and $N$ testing functions [1-8]. For electrically large problems $N$ becomes large, making an MoM analysis computationally prohibitive. To mitigate this limitation, the FMM and MLFMA have been developed [1,4-8], these employing an identity involving the free-space Green's function. Rather than directly computing order $N^{2}$ interactions, the FMM identity divides the basis and testing functions into "near" and "far" terms (the "far" terms need not be in the far field), with the relatively few "near" terms handled as in the traditional MoM. The "far" terms are evaluated by dividing the basis and testing functions into clusters, with interactions computed cluster by cluster. The MLFMA requires order $N \log N$ memory and $P N \log N$ computation time (CPU), where $P$ is the number of iterations required of the iterative solver (e.g. conjugate gradients [15]).

As mentioned, within the context of the FMM and MLFMA, the "near" terms are evaluated as in the traditional MoM. Therefore, for the half-space problem, the dyadic Green's function used for the "near" terms are evaluated via the complex-image technique [9], this demonstrated to be a highly efficient and accurate means of evaluating such terms. We have demonstrated previously, in the context of the "far" FMM terms, that the complex-image technique often yields slow convergence of the FMM identities [1,7]. Therefore, we seek an efficient and accurate means of evaluating the "far" FMM terms, with such compatible with the free-space Green's function, around which the FMM was originally designed. 
Assume the source and observation point are in the same half-space layer. The Sommerfeld integral of a typical dyadic-Green's-function component consists of a direct term plus a reflected term. The direct term corresponds to radiation from the source to the observer, as in a free-space region characteristic of the material properties in which the source and observer reside. This component of the Green's function corresponds to the free-space problem, and its Sommerfeld integral can be evaluated in closed form as the free-space Green's function [9]. This relationship is referred to as the Sommerfeld identity [9]. The complexity in evaluating the half-space Green's function is found in the reflected term, with a typical reflected component of the Sommerfeld integral expressed as

$$
I_{R}=\int_{-\infty}^{\infty} \Gamma\left(k_{z i}\right) \frac{k_{\rho}}{2 j k_{z i}} H_{o}^{(2)}\left(k_{\rho}|\rho-\rho|\right) e^{-j k_{z i}\left|z+z^{\prime}\right|} d k_{\rho}=\int_{-\infty}^{\infty} \Gamma\left(k_{z i}\right) F\left(k_{\rho}\right) d k_{\rho}
$$

where $\rho$ and $\rho^{\prime}$ represent the transverse observation and source points, respectively, with $z$ and $z^{\prime}$ representing the observation and source point along the $z$-direction (the half-space planar surface is normal to $z$ ). In (1) the source and observation point are both in region $i$, with region 1 at $z>0$ and region 2 at $z<0$, where $z=0$ corresponds to the air-soil interface. Finally, in (1) the $z$-directed wavenumber $k_{z i}=\left[k_{i}^{2}-k_{\rho}^{2}\right]^{1 / 2}$, where $k_{i}$ is the wavenumber for region $i$. The spectral reflection coefficient $\Gamma\left(k_{z i}\right)$ is a simple function of polarization, and is given in [16].

For large transverse distance between the source and observer, the Hankel function in (1) can be expressed in terms of its large-argument approximation, and the integral in (1) becomes

$$
I_{R} \approx \int_{-\infty}^{\infty} \Gamma\left(k_{z i}\right) \frac{k_{\rho}}{2 j k_{z i}} \sqrt{\frac{2}{k_{\rho}|\rho-\rho| \pi}} e^{-j\left(\left.k_{\rho}\right|^{\rho}{ }^{\prime} \mid-\pi / 4\right)} e^{-j k_{z i}\left|z+z^{\prime}\right|} d k_{\rho}
$$

The integral in (2) can be evaluated asymptotically via a steepest-descent path (SDP) analysis, with the saddle point $\mathrm{k}_{\rho s}$ defined by 


$$
\frac{k_{\rho s}}{\sqrt{k_{i}^{2}-k_{\rho s}^{2}}}=\frac{|\rho-\rho|}{\left|z+z^{\prime}\right|}
$$

with this having well-known physical meaning [10]. In the simple SDP evaluation of (2) we yield a result which is a function of the reflection coefficient $\Gamma\left(k_{z i}\right)$ evaluated for $k_{\rho}=k_{\rho s}$. To improve accuracy of the asymptotic result, we can employ a higher-order approximation to the reflection coefficient, in the vicinity of the stationary point, expressed as

$$
\left.\Gamma\left(k_{z i}\right) \approx \sum_{n=0}^{N} \frac{1}{n !} \frac{\partial^{n}}{\partial k_{z i}{ }^{n}} \Gamma\left(k_{z i}\right)\right|_{k_{z i}=k_{z i s}}\left(k_{z i}-k_{z i s}\right)^{n}
$$

where we take $N$ terms of the Taylor series, for $k_{z i}$ about the stationary point $k_{z i s}$. We therefore represent the reflection coefficient in the form

$$
\Gamma\left(k_{z i}\right) \approx \sum_{n=0}^{N} \Gamma_{n} k_{z i}^{n}
$$

where $\Gamma_{n}$ are constants computed from (4).

Note from (3) that the stationary point becomes singular when both the source and observer are near the interface located at $z=0$. For this case, we compute the stationary point numerically, by solving $\partial / \partial k_{\rho} F\left(k_{\rho}\right)=0$ (see (1)). We have found that a numerical search using Muller's method typically finds the stationary point in a small number of iterations (three or four).

\section{B. Far FMM interactions}

Returning to the integral in (1), we now have 


$$
\begin{aligned}
I_{R} & \approx \sum_{n=0}^{N} \Gamma_{m} \int_{-\infty}^{\infty} \frac{k_{\rho} k_{z i}^{n}}{2 j k_{z i}} H_{o}^{(2)}\left(k_{\rho}|\rho-\rho|\right) e^{-j k_{z i}\left|z^{+} z^{\prime}\right|} d k_{\rho} \\
& =\sum_{n=0}^{M} \Gamma_{n} \frac{\partial^{n}}{( \pm j)^{n} \partial z^{n}} \int_{-\infty}^{\infty} \frac{k_{\rho}}{2 j k_{z i}} H_{o}^{(2)}\left(k_{\rho}\left|\rho-\rho^{\prime}\right|\right) e^{-j k_{z i}\left|z^{\prime} z^{\prime}\right|} d k_{\rho} \\
& =\sum_{n=0}^{N} \Gamma_{n} \frac{\partial^{n}}{( \pm j)^{n} \partial z^{n}} \frac{e^{-j k_{i} R_{r}}}{R_{r}}
\end{aligned}
$$

where the plus sign corresponds to source and observation points at $z<0$, with the minus sign corresponding to $z>0$. To reach the bottom equation in (6), we have utilized the Sommerfeld identity [9], and $R_{r}$ is the distance from an equivalent real image point to the observer. In previous work, concerning treatment of the far FMM terms for the half-space problem, only the $n=0$ term was utilized, resulting in a single real image with weight $\Gamma_{\mathrm{o}}[1,7]$. Here we utilize higher-order $N>0$ terms, resulting in improved accuracy, while still retaining a form appropriate for FMM implementation.

The fundamental FMM identities are [4-8]

$$
\begin{aligned}
& \frac{e^{-j k_{i}|\boldsymbol{x}-\boldsymbol{x}|}}{|\boldsymbol{x}-\boldsymbol{x}|} \approx \frac{-j k_{i}}{4 \pi} \iint_{4 \pi} e^{-j k_{i} \hat{\boldsymbol{k}} \cdot\left(\boldsymbol{x}-\boldsymbol{x}_{m}\right)} T_{L}\left(k_{i} X_{m^{\prime} m}, \hat{\boldsymbol{k}} \cdot \hat{\boldsymbol{X}}_{m^{\prime} m}\right) e^{+j k_{i} \hat{\boldsymbol{k}} \cdot\left(\boldsymbol{x}^{\prime}-\boldsymbol{x}_{m^{\prime}}\right)} d^{2} \hat{\boldsymbol{k}} \\
& T_{L}\left(k_{i} X_{m^{\prime} m}, \hat{\boldsymbol{k}} \cdot \hat{\boldsymbol{X}}_{m^{\prime} m}\right)=\sum_{l=0}^{L}(-j)^{l}(2 l+1) h_{l}^{(2)}\left(k_{i} X_{m^{\prime} m}\right) P_{l}\left(\hat{\boldsymbol{k}} \cdot \boldsymbol{X}_{m^{\prime} m}\right)
\end{aligned}
$$

where $\boldsymbol{x}$ and $\boldsymbol{x}^{\prime}$ denote the observation and source points, respectively, $\boldsymbol{x}_{m}$ and $\boldsymbol{x}_{m}{ }^{\prime}$ denote the source and observation FMM groups, $\boldsymbol{X}_{m m}$ is a vector (of magnitude $X_{m m}$ ) connecting the source and observation groups, $h_{l}^{(2)}$ is a spherical Hankel function, and $P_{l}$ is a Legendre polynomial [4-8]. In (7a) we integrate over the surface of a unit sphere, with unit normal $\hat{\boldsymbol{k}}$. We see that each of the terms 
in the final representation of (6) are in a form compatible with (7). In particular, we utilize the relation from $(7 \mathrm{a})$ that

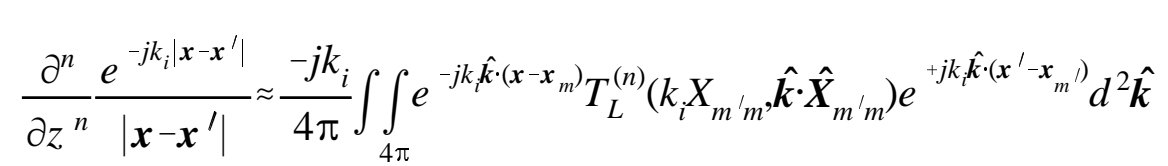

$$
\begin{aligned}
& T_{L}^{(n)}\left(k_{i} X_{m^{\prime} m}, \hat{\boldsymbol{k}} \cdot \hat{\boldsymbol{X}}_{m^{\prime} m}\right)=\left(-j k_{i} \hat{k}_{z}\right)^{n} T_{L}\left(k_{i} X_{m^{\prime} m}, \hat{\boldsymbol{k}} \cdot \hat{\boldsymbol{X}}_{m^{\prime} m}\right)
\end{aligned}
$$

where $\hat{k}_{z}=\hat{\boldsymbol{k}} \cdot \hat{z}$, with $\hat{z}$ representing a $z$-directed unit vector. Therefore all terms in the last form of (6) are directly applicable to the FMM identities, with the minimal modification in (8), thereby realizing a simple extension of the free-space FMM to the half-space case. A similar analysis can be carried out for the general case of layered media.

\section{Singularity extraction}

The principal utility of the procedure developed in (8) is for the case in which the target of interest touches or is very near the half-space interface, since for this case the simpler evaluation [1] in which we simply utilize the $n=0$ term (a single real image with appropriate amplitude) is inappropriate. When the target is near to or touching the interface, the real-image locations may be close to or even touching some of the testing functions on the target surface. This issue is important for both the "near" and "far" terms in the MLFMA model. We therefore emphasize the need to perform careful singularity extraction when performing such (image source)-(testing function) evaluations, to avoid numerical complications. Related singularity-extraction formulations have been used for many years in the context of MoM near-term interactions [11].

As discussed above, when the source and observation point are in the same half-space region, each component of the half-space Green's function is composed of two terms. One term represents direct radiation as if in a free-space medium, and the other term represents reflection at the half-space interface. For the former term, interactions between proximate expansion and testing functions are handled as in previous free-space scattering problems [11]. We direct special attention toward the reflection term, this particularly important when using image sources, as discussed in the previous 
section. Performing singularity extraction on the reflection terms, we must compute integrals of the form

$$
\begin{gathered}
I_{1}=\int_{S}\left[\frac{e^{-j k_{i} R_{p}}}{R_{p}}-\frac{1}{R_{p}}\right] d S^{\prime}+\int_{S} \frac{1}{R_{p}} d S^{\prime} \\
I_{3}=\int_{S}\left[\frac{1+j k_{i} R_{p}}{R_{p}^{3}} e^{-j k_{i} R_{p}}-\frac{1+k_{i}^{2} R_{p}^{2} / 2}{R_{p}^{3}}\right]\left(\boldsymbol{X}_{p}-\boldsymbol{X}^{\prime}\right) d S^{\prime}+\int_{S} \frac{1+k_{i}^{2} R_{p}^{2} / 2}{R_{p}^{3}}\left(\boldsymbol{X}_{p}-\boldsymbol{X}^{\prime}\right) d S^{\prime}
\end{gathered}
$$

where $\boldsymbol{X}^{\prime}$ is a vector locating the source point, $\boldsymbol{X}_{p}$ locates a vertex on the source patch basis function (see Fig. 1), and $R_{p}=\left[\left(x-x^{\prime}\right)^{2}+\left(y-y^{\prime}\right)^{2}+\left(z+z^{\prime}+b\right)^{2}\right]^{1 / 2}$. When applying the complex-image technique, the constant $b$ is in general imaginary, while $b=0$ for the asymptotic representation introduced in Sec. IIB. In the latter case the integrals in (9) are evaluated as in the free-space treatment of MoM near terms. Special attention is required with regard to the complex-image technique, since $b$ is now in general imaginary. We introduce the new distance $R_{q}=\left[\left(x-x^{\prime}\right)^{2}+\left(y-y^{\prime}\right)^{2}+\left(z+z^{\prime}\right)^{2}-2\left(z_{\text {min }}^{\prime}+z\right) b+b^{2}\right]^{1 / 2}$, where $z_{\text {min }}^{\prime}$ represents the minimum distance between a source point and the interface, this corresponding to the most singular portion of the integral. We now modify (9) as

$$
\begin{gathered}
I_{1}^{\prime}=\int_{S}\left[\frac{e^{-j k_{i} R_{p}}}{R_{p}}-\frac{1}{R_{q}}\right] d S^{\prime}+\int_{S} \frac{1}{R_{q}} d S^{\prime} \\
I_{2}^{\prime}=\int_{S}\left[\frac{\boldsymbol{X}_{p}-\boldsymbol{X}^{\prime}}{R_{p}} e^{-j k_{i} R_{p}}-\frac{\boldsymbol{X}_{p}-\boldsymbol{X}^{\prime}}{R_{q}}\right] d S^{\prime}+\int_{S} \frac{\boldsymbol{X}_{p}-\boldsymbol{X}^{\prime}}{R_{q}} d S^{\prime}
\end{gathered}
$$




$$
I_{3}^{\prime}=\int_{S}\left[\frac{1+j k_{i} R_{p}}{R_{p}^{3}} e^{-j k_{i} R_{p}}-\frac{1+k_{i}^{2} R_{q}^{2} / 2}{R_{q}^{3}}\right]\left(\boldsymbol{X}_{p}-\boldsymbol{X}^{\prime}\right) d S^{\prime}+\int_{S} \frac{1+k_{i}^{2} R_{q}^{2} / 2}{R_{q}^{3}}\left(\boldsymbol{X}_{p}-\boldsymbol{X}^{\prime}\right) d S^{\prime}
$$

The integrals in (10) are now in a form appropriate for previous MoM singularity-extraction studies [11]. Summarizing, we have employed singularity extraction for the image terms near or touching testing functions. The asymptotic-based MLFMA "far" terms are handled analogous to previous MoM singularity-extraction techniques, with a small modification required for the "near" MLFMA terms modeled using a complex-image analysis of the half-space Green's function.

\section{Simple Preconditioner}

The solution to an integral equation yields a matrix equation [1-8] of the form $\boldsymbol{Z i}=\boldsymbol{v}$, where $\boldsymbol{Z}$ is an $N \times N$ matrix (for $N$ unknowns), $\boldsymbol{i}$ is an $N \times 1$ vector representing the unknown basis-function coefficients, and $v$ is an $N \times 1$ vector representing the driving function. When solving this equation via the MLFMA, we do not explicitly fill the matrix $\boldsymbol{Z}$, but rather efficiently compute the matrix product $\boldsymbol{Z} \boldsymbol{i}$ with order $N \log N$ complexity $[1,4,-8]$. Let $\boldsymbol{P}$ be an $N \times N$ matrix, with the goal that the

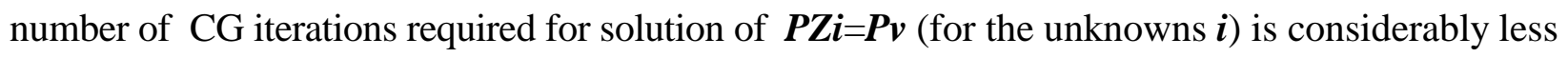
than that required for $\boldsymbol{Z} \boldsymbol{i}=\boldsymbol{v}$. As we discuss further below, this implies that the condition number of $\boldsymbol{P Z}$ must be considerably smaller than that of $\boldsymbol{Z}[17,18]$. We have the attendant requirement that implementation of this preconditioner be efficient.

We desire the matrix product $\boldsymbol{P Z}$ to be highly diagonal, thereby improving its condition number [6,7]. Consider the $n$th basis function $\boldsymbol{b}_{n}$ (corresponding to the $n$th element in $\boldsymbol{i}$ ), and let the set $S=\left\{\boldsymbol{b}_{n}{ }^{(1\}}, \boldsymbol{b}_{n}{ }^{(2)}, \ldots, \boldsymbol{b}_{n}{ }^{(K)}\right\}$ contain all $K$ testing functions within a prescribed distance of $\boldsymbol{b}_{n}$, where $\boldsymbol{b}_{n}^{(1)}=\boldsymbol{b}_{n}$, assuming a Galerkin procedure [1-8]. We now solve the following (typically small) matrix equation 


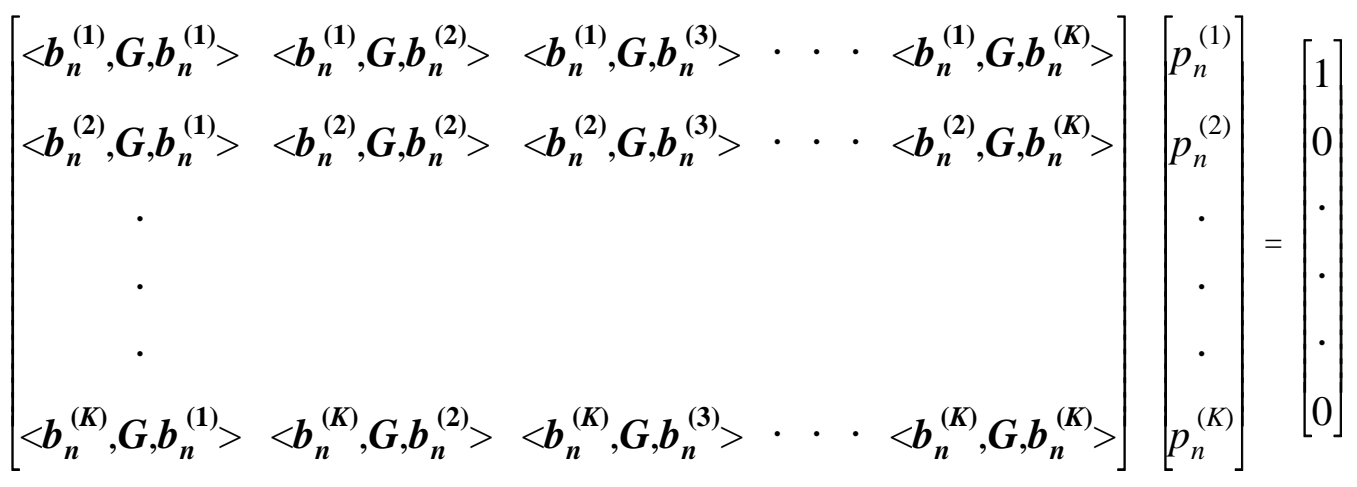

In (11) the three components in the inner product $\left\langle\boldsymbol{b}_{n}{ }^{(i)}, \boldsymbol{G}, \boldsymbol{b}_{n}{ }^{(j)}\right\rangle$ are, left to right, the $i$ th basis function, the appropriate Green's function, and the $j$ th testing function [1-8] (all expansion and testing functions are in $S$ ). The components $p_{n}{ }^{(1)}, p_{n}{ }^{(2)}, \ldots, p_{n}{ }^{(K)}$ are defined to constitute the $K$ non-zero elements in the $n$th row of $\boldsymbol{P}$, with $K<<N$ typically. Specifically, coefficient $p_{n}{ }^{(k)}$ is placed in the $n$th row of $\boldsymbol{P}$, at the position corresponding to basis function $\boldsymbol{b}_{n}{ }^{(k)}$. Note, however, in practice we do not explicitly fill the (large) matrix $\boldsymbol{P}$, but rather perform the matrix product $\boldsymbol{P}(\boldsymbol{Z i})$ directly, with $\boldsymbol{Z i}$ computed efficiently via the MLFMA. Using the above procedure, the element $(n, n)$ of $\boldsymbol{P Z}$ is unity, while the positions corresponding to the other basis functions in $S$, in the $n$th row of $\boldsymbol{P Z}$, are zero. With regard to the remaining terms in the $n$th row of $\boldsymbol{P Z}$, the (expansion function)-(testing function) interactions not accounted for in $S$ are relatively small due to the relative distance between these functions. Consequently, this procedure, applied to all rows of $\boldsymbol{P Z}$, yields a highly diagonal matrix, leading to improved convergence of $\boldsymbol{P Z} \boldsymbol{i}=\boldsymbol{P} \boldsymbol{v}, v i s-\grave{a}$-vis $\boldsymbol{Z} \boldsymbol{i}=\boldsymbol{v}$.

Considering the added computational complexity associated with implementing the above preconditioner, note that we need solve $N$ small matrix equations of the form in (11), each of which yields the respective non-zero elements in each row of $\boldsymbol{P}$. Hence, the additional computational burden is of order $N$ complexity, with this a modest escalation from the $N \log N$ MLFMA. With regard to additional memory needs, we only store the approximately $N K$ non-zero elements in $\boldsymbol{P}$ (approximate because each row of $\boldsymbol{P}$ need not require the same number $K$ ). In the results reported below, we apply the procedure in (11) to all testing functions within a half wavelength of the $n$th basis function, and therefore $K$ is typically on the order of ten. 


\section{Example Results}

\section{A. Green's-function analysis}

In Sec. II we developed a simple technique by which the dyadic half-space Green's function can be evaluated efficiently and accurately, within the context of the FMM or MLFMA. As indicated in Sec. II, in our FMM/MLFMA model [1,7] the "near" terms employ complex-image-technique evaluation of the half-space Green's function, this having the advantage that the subsequent Green's function representation is only a function of the source distance from the half-space interface (assuming the source and observer are in the same layer [9]). While this approach is useful within the context of the FMM/MLFMA, note that the stationary-point in (4) is a function of both the source and observation point (not just the source height), with this issue discussed further below, when we address its use in the half-space FMM/MLFMA.

It is of interest to use the technique presented in Sec. II to compute example components of the half-space Green's function, with comparison to a direct numerical evaluation of the Sommerfeld integral (performed along the real $k_{\rho}$ axis, using Gauss-quadrature integration). We consider Green'sfunction components $G_{A x x}$ and $G_{A z z}$, with the reader referred to [16] for more details on the Green'sfunction. In Fig. 2 we present results for these Green's-function components, for the source and observer $0.1 \lambda$ wavelengths from the half-space interface (vertical height), as a function of variable lateral separation. The half-space is nonmagnetic, and the region in which the source and observer reside is characterized by relative permittivity $\epsilon_{r}=1.0$ while the other half-space region is characterized by $\epsilon_{r}=5.0-j 0.2$. In Fig. 2 we present three sets of results, the procedure of Sec. II with $N=0$ (single real image [1,7]), $N=4$ (higher-order asymptotics), and a real-axis numerical integration of the Sommerfeld integral. We see that the higher-order asymptotic solution provides virtually exact results, down to a lateral separation of $0.7 \lambda$. The single-real-image result yields agreement to a typical error of less than $15 \%$. The $N=0$ solution improves markedly as the height of the source and observer are increased. Although not shown here, the agreement between the higher-order asymptotic solution and direct Sommerfeld integration remains highly accurate even when both the source and observer are $o n$ the interface, while the real-image solution fails entirely (for reasons discussed after 
$(5))$.

\section{B. MLFMA validation}

We have modified our MLFMA software [1], utilizing the method in Sec. II to handle the reflected dyadic components, for "far" MLFMA cluster-cluster interactions. As we demonstrate, the model is now applicable to the target sitting on the half-space interface. In this context the following comments are important. In the MoM model and for the "near" MLFMA terms, the complex-image technique is utilized for evaluation of the half-space Green's function. If a source point (basis function) is near the interface, the associated complex images will be so as well [9]. Therefore, we have found that one must be careful in utilizing well-know techniques for singularity extraction [11], when performing the (expansion function)-(testing function) inner products. Not only must one perform singularity extraction for the traditional real source points, when employing nearby testing functions [11], one must also do the same for complex-image sources near the interface, when employing near-interface testing functions (see Sec. IIC). Such procedures have been implemented in our MoM model, and for the "near" MLFMA interactions.

Concerning the "far" MLFMA terms employing the representation in (4), we noted in Sec. IVA that the stationary point is a function of the source and observation positions, implying a different representation for each (basis function)-(testing function) interaction. To simplify the implementation, within the context of "far" MLFMA interactions, the stationary point is defined by the center positions of the source and observation clusters. Therefore, for a given cluster-cluster interaction, all associated expansion and testing functions utilize the same stationary point. This is clearly an approximation, being more accurate for smaller cluster sizes, but comparisons with a rigorous MoM solution suggests this approximation yields accurate results.

To validate the procedures developed in Sec. II, we consider a dielectric cylinder of $60 \mathrm{~cm}$ diameter and $2.5 \mathrm{~m}$ height placed on a dielectric half space characterized by conductivity $\sigma=0.01 \mathrm{~S} / \mathrm{m}$ and complex relative permittivity $\epsilon_{r}=5-\mathrm{j} 0.2$. The cylinder is in direct contact with the half-space interface. The dielectric constant of the lossless cylinder is $\epsilon_{r}=3.2$ and the operating frequency is 
$f=300 \mathrm{MHz}$. This is a relatively small problem, employing $N=6,060$ unknowns, allowing a direct comparison between a MLFMA and MoM analysis. In the MoM analysis all Green's function computations are performed via the complex-image technique, thereby providing validation of the MLFMA in which we employ an approximation to the Green's function in the context of the "far" terms (Sec. II). The bistatic RCS from this target is shown in Fig. 3, for an incident angle of $\theta_{i}=45^{\circ}$ and $\phi_{i}=0^{\circ}$, and the scattered fields are observed for $\phi_{s}=0^{\circ}$ and $0^{\circ} \leq \theta_{s} \leq 90^{\circ}$. It is seen that the agreement between the MoM and MLFMA results is virtually exact, where here we employ three MLFMA levels. We have found similar agreement between the MoM and MLFMA solutions for all targets we have examined, for both conducting and dielectric targets. The computations were performed on a $450 \mathrm{MHz}$ Pentium III computer, and with the MoM and MLFMA requiring respectively 930 and 410 Mbytes of RAM (memory), and 1130 and 372 minutes of CPU time.

\section{Preconditioner}

In Sec. III we presented a preconditioner, which we now examine in the context of example MLFMA results. We present results for a trihedral scatterer, composed of infinitesimally thin perfect conductors. In a rectangular coordinate system, with $z=0$ corresponding to the air-ground interface, the three constitutive triangles have vertices positioned as follows, in units of meters: triangle one $(-4,4,0.2),(0,0,0.2),(0,0,4.2)$; triangle two $(-4,4,0.2),(4,4,0.2),(0,0,0.2)$; and triangle three $(0,0,0.2)$, $(0,0,4.2)$ and $(4,4,0.2)$. The soil is modeled as a lossy half space, with parameters characteristic of Yuma soil with 5\% water content [19].

In Fig. 4 we present a comparison of the number of iterations required to achieve convergence of the conjugate-gradient (CG) iterative solver, for an error of $0.1 \%$, with the error defined as $\|\boldsymbol{P z i}-\boldsymbol{P} \boldsymbol{v}\| /\|\boldsymbol{P} \boldsymbol{v}\|$ (see Sec. III). Results are shown as a function of $N$, with the variability in $N$ corresponding to covering a frequency range of $40-200 \mathrm{MHz}$. We consider plane-wave incidence at the angles $\phi=0^{\circ}$ and $\theta=45^{\circ}$ (in the coordinate system of the trihedral, defined above), in vertical polarization. In Fig. 4 we present CG convergence results when there is no preconditioner, a diagonal preconditioner [6], a block-diagonal preconditioner [6], and the preconditioner reviewed in Sec. III (termed the MN preconditioner, adopting the nomenclature of $[13,14])$. The diagonal preconditioner 
uses a diagonal $\boldsymbol{P}$ equal to the inverse of the diagonal of $\boldsymbol{Z}$, while the block-diagonal preconditioner is similarly applied to the inverse of diagonal blocks of $\boldsymbol{Z}$ (here the block size is equal to the MLFMA clusters at the finest level [6]). We see that the MN preconditioner typically requires 80 fewer CG iterations than the other preconditioners, with the block-diagonal preconditioner being particularly unstable as the number of unknowns $N$ increases. These computations have been performed using the widely used triangular-patch basis functions [11], with ten basis functions per wavelength.

It is also of interest to examine the additional computational complexity of the MN preconditioner. In Fig. 5 we plot the CPU time per CG iteration (in seconds), for the four cases examined in Fig. 4. The absolute numbers required per iteration is machine dependent (here we used a $500 \mathrm{MHz}$ Pentium III PC with 1 GByte of memory), and therefore the principal message of Fig. 5 is that the $\mathrm{MN}$ preconditioner (as well as the other preconditioners) requires virtually no additional CPU time, relative to applying no preconditioner at all.

It is well known that the EFIE condition number is a strong function of the basis-function sample density $[17,18]$. We consider the convergence properties of the preconditioners considered in Fig. 4, as a function of the triangular-patch basis function sample density. In particular, we consider a $7 \mathrm{~m} \times 7 \mathrm{~m} \times 8 \mathrm{~m}$ trihedral over soil, as in the above examples. The target is represented by $N=11,175$ unknowns, and we consider plane-wave excitation over the frequency range 150-300 $\mathrm{MHz}$, corresponding to a sample density ranging from 7.2 to 15 basis functions per wavelength. We see in Fig. 6 that the utility of the MN preconditioner is clearly manifested as the sample density increases, mitigating some of the EFIE shortcomings. These MLFMA computations were computed to a $1 \% \mathrm{CG}$ error, and the number of MLFMA levels ranged from 3 to 5, over the bandwidth considered.

In Figs. 4 and 6 the preconditioners have been compared in terms of the number of iterations required for $\mathrm{CG}$ convergence, to a specified accuracy. As the number of unknowns $N$ becomes large, the computation time required for each CG iteration increases as $N \log N$, for the MLFMA. Consequently, the improved CG convergence reported in Figs. 4 and 6 is substantial in terms of 
overall computation time. It is important, however, to demonstrate that the condition number of the matrix product $\boldsymbol{P Z}$ is indeed reduced, vis-à-vis that of the original matrix $\boldsymbol{Z}$. Using a standard $l^{2}$ norm, the condition number is equal to the ratio of the largest to the smallest singular values, computed via a singular-value decomposition (SVD) [15]. We have found that the MN preconditioner yields a condition number that is consistently a factor-two smaller than that of the original matrix $\boldsymbol{Z}$, as well as that with the diagonal preconditioner. The condition number of the block-diagonal preconditioner is far worse than that of the other examples, consistent with its poor CG convergence shown in Figs. 4 and 6.

We have employed the MN preconditioner in the computation of all MLFMA results presented in this paper. For the examples considered, we have found that the CG convergence is typically accelerated by a factor of two, vis-à-vis using no preconditioner.

\section{Tree-like target}

Having validated the MLFMA and discussed the preconditioner, we now present several example results of interest to remote-sensing applications. In the first example we consider the treelike target depicted in Fig. 7, where in this figure we plot the induced electric current on the surface of the dielectric target at $500 \mathrm{MHz}$, with the tree-like target above Yuma soil with $5 \%$ water content [19]. The tree height is nominally $6 \mathrm{~m}$, the diameter of the main trunk is $50 \mathrm{~cm}$, and in this example the electrical properties of the tree are $\epsilon_{r}=4$ and $\sigma=0.01 \mathrm{~S} / \mathrm{m}$. These parameters are not necessarily representative of a real tree, and are simply selected for the purpose of a numerical example. The MLFMA model is applicable to general tree properties (here assuming for simplicity the internal tree electrical properties are homogeneous and isotropic), and the study of actual trees will be the subject of future research. For the computations responsible for Fig. 7 N=62,538 unknowns and six MLFMA levels are employed. Computation of these results required 15.6 hours of CPU on a 300 Silicon Graphics computer, using 4.6 Gbytes of RAM.

For foliage-penetrating radar applications, one of the principal sources of clutter involves dihedral-like scattering at the interface of the soil and tree trunk. At the VHF and low UHF 
frequencies of interest, the effects of the leaves and small branches are of less importance. It is of interest to examine the RCS of a tree-like target like that in Fig. 7 vis-à-vis the RCS when the branching structure is not present (i.e., to compare the RCS of the target in Fig. 7 to the RCS of a simple cylinder target of the same properties, ignoring the branches). In Fig. 8 we compare the monostatic RCS of the tree-like target to that of a cylinder, at frequencies of 50, 100 and $300 \mathrm{MHz}$, for incidence angles $\theta_{i}=45^{\circ}$ and $-180^{\circ} \leq \phi_{i} \leq 180^{\circ}$. As is expected, the rate of variation in the scattered fields with azimuthal angle quickens as the frequency of operation increases. The average of the RCS with angle for the tree-like target tends to settle about the isotropic response from the associated cylinder, although the variation can be substantial. For the example considered it appears that the effects of the tree are more substantial in VV polarization, vis-à-vis $\mathrm{HH}$, although this is an issue that warrants more-careful consideration.

\section{E. Tank}

The MLFMA software we have developed is applicable to conducting or dielectric targets on a half space. As an example of model utility for conducting targets, we consider scattering from a tank situated atop Yuma soil with 5\% water content [19]. In Fig. 9 we present the induced currents on the surface of the tank at $500 \mathrm{MHz}$, this corresponding to $\mathrm{V}$ and $\mathrm{H}$ polarization and incidence at the angles $\theta_{i}=45^{\circ}$ and $\phi_{i}=60^{\circ}$ (see coordinate system in figure). For these computations the target is characterized by $N=46,131$ unknowns and the MLFMA employed five levels.

As a further demonstration of the model utility, in Fig. 10 we present example SAR imagery for the tank, for $\mathrm{HH}$ polarization, at a depression angle of $\theta_{i}=55^{\circ}$, employing a $60^{\circ} \mathrm{SAR}$ aperture, with results in this figure shown for two target orientations relative to the linear synthetic aperture (the aperture is parallel to the horizontal axes in these figures). When computing a SAR signature one must compute multiple scattered waveforms, as a function of frequency and angle of incidence. Therefore, in these computations the bandwidth considered is $50-200 \mathrm{MHz}$, in $4 \mathrm{MHz}$ increments. 


\section{F. Rough surface}

In computation of scattering from a rough surface, truncating the surface constitutes an important issue. In most previous studies the surface has been truncated abruptly, and the effects of the surface truncations are mitigated by employing beam excitation [12]. This approach generally works well, although one must be careful that surface waves are not excited strongly, since these will propagate away from the illuminated region and interact with the surface truncation. In the context of remote-sensing applications, one often operates over a wide frequency range. The required beamwidth for beam excitation is a function of frequency, becoming very large at low frequencies (such as the $50 \mathrm{MHz}$ computations employed in Fig. 10). Therefore, it is of interest to consider an alternative truncation methodology for computing rough-surface scattered fields. The formulation presented in Sec. II allows target placement in direct contact with the air-soil interface of a half space. We therefore use a three-dimensional closed surface to represent surface roughness (see Fig. 11). The bottom of this surface is flat and is in direct contact with the half space, while the top portion of the surface invokes the roughness of interest. A slow taper is employed at the ends of the surface, constituting a transition from the half space to the roughness. The electrical properties of the soil half space are made identical to those of the aforementioned closed dielectric target. We employ plane-wave excitation, thereby mitigating the problems of beam excitation over a large bandwidth.

The rough-surface scattering problem is typically treated statistically, for which a large ensemble of scattered waveforms are required to assess scattering from the stochastic surface. Such a careful analysis of rough-surface scattering is beyond the scope of the present study, and therefore we present results for an example rough but deterministic surface. The height of the threedimensional surface is characterized

$$
h(x, y)=\left[1+\sin \left(2 \pi n x / x_{m}\right)\right]\left[1+\sin \left(2 \pi n y / y_{m}\right)\right]
$$

where $x_{m}=3.13 \mathrm{~m}$ and $y_{m}=3.13 \mathrm{~m}$ are the widths of the surface in the two dimensions, and $n=4$ in this example (see Fig. 12). The electrical properties of the half-space subsurface and of the flush dielectric target are given by $\epsilon_{r}=5-j 0.2$ and $\sigma=0.01 \mathrm{~S} / \mathrm{m}$. The frequency of operation is $f=300 \mathrm{MHz}$, 
and therefore the rough surface constitutes $7 \times 7$ wavelengths in the dielectric. A total of $N=13,848$ unknowns are employed, utilizing four MLFMA levels. Example results are shown in Fig. 13, for incidence at $\theta_{i}=45^{\circ}$ and $\phi_{i}=0^{\circ}$, and the bistatic RCS is viewed for $0^{\circ} \leq \theta_{s} \leq 90^{\circ}$ and $\phi_{s}=0^{\circ}$.

\section{Conclusions}

The MLFMA has been extended to the problem of a general conducting or dielectric target in direct contact with the interface of a lossy half space. The reflection term in the spectral representation of the half-space Green's function has been evaluated using a higher-order asymptotic analysis, yielding a relatively simple extension of our previous first-order saddle-point analysis [1]. A careful investigation has been presented to confirm the accuracy of this Green's function analysis, followed by a series of example results from general conducting and dielectric targets. In all of these numerical examples we have found that the initial integral-equation formulation yields slow convergence of the conjugate-gradient (CG) iterative matrix solver employed in the context of the MLFMA. Therefore, we have also directed attention toward development of a simple, improved preconditioner. The $\mathrm{MN}$ preconditioner has been employed, it developed originally in the mathematics community $[13,14]$. We have found that this simple preconditioner requires minimal additional computational expense, and it typically accelerates CG convergence by a factor of two, for both conducting and dielectric targets.

We have presented example results for scattering from a tree-like target, a tank and from a rough air-soil interface. In the latter example, the rough surface is modeled as a closed dielectric target in direct contact with the lossy half space, yielding a smooth transition between the infinite dielectric interface and the finite rough surface. In the results presented here we considered a deterministic rough surface, for simplicity, and in future studies we will employ this formulation to address scattering from a stochastic dielectric rough surface. In addition, future studies will focus on the analysis of scattering from a composite target, composed of dielectric and conducting components. For example, in the present study we have addressed scattering from a tank and from a tree-like target in the presence of a half space, with each target in isolation. In the future this analysis will be extended to consider scattering from a conducting target (e.g. a tank) in the presence 
of foliage.

\section{References}

[1] N. Geng, A. Sullivan and L. Carin, "Multi-level fast-multipole algorithm for scattering from conducting targets above or embedded in a lossy half space," IEEE Trans. Geoscience Remote Sensing, vol. 38, pp. 1567-1579, July 2000.

[2] J. He, T. Yu, N. Geng and L. Carin, "Method-of-moments analysis of electromagnetic scattering from a general three-dimensional dielectric target embedded in a multi-layered medium," Radio Science, vol. 35, pp. 305-313, Mar.-Apr. 2000.

[3] N. Geng and L. Carin, "Ultra-wideband, short-pulse scattering from a dielectric body of revolution buried in a lossy, dispersive layered medium," IEEE Trans. Antennas Propagat., vol. 47, pp. 610-619, April 1999.

[4] R. Coifman, V. Rokhlin, S. Wandzura, "The fast multiple method for the wave equation: a pedestrian prescription”, IEEE Ant. and Prop. Magazine, vol. 35, pp.7-12, June 1993.

[5] J.M. Song, W.C. Chew, "Multilevel fast multiple algorithm for solving combined field integral equations of electromagnetic scattering", Micr. Opt. Techn. Lett., vol. 10, pp. 14-19, Sept. 1995.

[6] J.M. Song, C.C. Lu, W.C. Chew, "Multilevel fast multiple algorithm for electromagnetic scattering by large complex objects", IEEE Trans. Ant. and Prop., vol. 45, pp. 1488-1493, Oct. 1997. [7] N.Geng, A. Sullivan, L. Carin, "Fast Multiple method for scattering from 3D PEC target situated in a half space environment”, Micr. Opt. Techn. Lett., vol.21, pp.399-405, June 1999.

[8] X.Q. Sheng, J.M. Jin, J. Song, C.C. Lu, W.C. Chew, "Solution of combinded field integral equation using multilevel fast multiple algorithm for scattering by homogeneous bodies", IEEE Trans. Ant. and Prop., vol. 46, pp. 1718-1726, Nov. 1998.

[9] Y.L. Chow, J.J. Yang, D.G. Fang, and G.E. Howard, “A closed-form spatial Green's function for the thick microstrip substrate", IEEE Trans. Micr. Theory and Techn., vol.39, pp. 588-592, March 1991.

[10] L.B. Felsen and N. Marcuvitz, Radiation and Scattering of Waves, IEEE Press, 1996, Ch. 4. [11] S.M. Rao, D.R. Wilton, A.W. Glisson, "Electromagnetic scattering from surfaces of arbitrary shape", IEEE Trans. Ant. and Prop., vol. 30, pp. 409-418, May 1982. 
[12] R.T. Marchand and G.S. Brown, "On the use of finite surfaces in the numerical prediction of rough surface scattering,"IEEE Trans. Ant. and Prop., vol. 47, pp. 600-604, April 1999.

[13] S.A. Vavasis, "Preconditioning for boundary integral equations," SIAM J. Matrix Anal. A, vol 13, pp. 905-925, July 1992.

[14] K. Chen, "On a class of preconditioning methods for dense linear systems from boundary elements," SIAM J. Sci. Comput., vol. 20, pp. 684-698, Sept. 1998.

[15] G.H. Golub and C.F. Van Loan, Matrix Computations, 3rd edition, Johns Hopkins Univ Press, 1996.

[16] K.A. Michalski and D.Zheng, "Electromagnetic scattering and radiation by surfaces of arbitrary shape in layered media, Part I and II," IEEE Trans. Ant. and Prop., vol.38, pp. 335-352, March 1990 [17] G.C. Hsiao and R.E. Kleinman, "Mathematical foundations for error estimation in numerical solutions of integral equations in electromagnetics," IEEE Trans. Antennas Prop., vol. 45, pp. 316-328 Mar. 1997.

[18] F.X. Canning and J.F. Scholl, "Diagonal preconditioners for the EFIE using a wavelet basis," IEEE Trans. Antennas Prop., vol. 44, pp. 1239-1246, Sept. 1996.

[19] L. Carin, N. Geng, M. McClure, J. Sichina, and L. Nguyen, "Ultra-wide-band synthetic-aperture radar for mine-field detection," IEEE Antennas Propag. Mag., vol. 41, pp. 18-33, Feb. 1999. 


\section{Figure Captions}

Figure 1. Schematic of an image expansion (here below the half-space interface) and a testing function (above the interface). The vectors $\boldsymbol{X}$ and $\boldsymbol{X}^{\prime}$ locate observation and source points, and $\boldsymbol{X}_{p}$ locates a vertex on the image basis function.

Figure 2. Component of the half-space dyadic Green's function, for both the source and observer $0.1 \lambda$ above a half-space characterized by $\epsilon_{r}=5.0-\mathrm{j} 0.2$, as a function of the lateral source-observer separation. Three results are shown: a simple real-image approximation $(N=0)$, a higher-order reflection coefficient representation (here with $N=4$ ), and a direct real-axis numerical integration of the Sommerfeld integral. Real and imaginary components shown separately. (a) $G_{x x}$, (b) $G_{z z}$

Figure 3. Bistatic RCS of a dielectric cylinder of $60 \mathrm{~cm}$ diameter and $2.5 \mathrm{~m}$ height placed on a dielectric half space characterized by conductivity $\sigma=0.01 \mathrm{~S} / \mathrm{m}$ and complex relative permittivity $\epsilon_{r}=5$-j0.2, with the dielectric constant of the lossless cylinder $\epsilon_{r}=3.2$, and operating frequency $f=300$ MHz. The incident angles are $\theta_{i}=45^{\circ}$ and $\phi_{i}=0^{\circ}$, and the scattered fields are observed for $\phi_{s}=0^{\circ}$ and $0^{\circ} \leq \theta_{s} \leq 90^{\circ}$

Figure 4. Number of CG iterations for solution of the MLFMA, for scattering from a trihedral scatterer over a lossy dispersive half space (soil), as a function of the number of unknowns $N$ (corresponding to varying the frequency from 40-200 MHz). Results are presented for the case of no preconditioner, the MN preconditioner (see Sec. III), a diagonal preconditioner, and a blockdiagonal preconditioner.

Figure 5. Computation time per CG iteration, for the example considered in Fig. 4.

Figure 6. Convergence of the MLFMA for a $7 \mathrm{~m} \times 7 \mathrm{~m} \times 8 \mathrm{~m}$ trihedral over soil. The number of unknowns is fixed at $N=11,175$ and the frequency is varied from 150-300 MHz, providing an examination of $\mathrm{CG}$ convergence as a function of basis-function density per wavelength. We consider the three preconditioners (plus no preconditioner) examined in Fig. 4.

Figure 7. Induced electric current on the surface of a tree-like dielectric target at $500 \mathrm{MHz}$, with the target atop Yuma soil with 5\% water content [19]. The tree height is nominally $6 \mathrm{~m}$, the diameter of the main trunk is $50 \mathrm{~cm}$, and in this example the electrical properties of the tree are $\epsilon_{r}=4$ and $\sigma=0.01 \mathrm{~S} / \mathrm{m}$.

Figure 8. Monostatic RCS from the tree-like target considered in Fig. 7, with comparison to the 
(isotropic) RCS of an identical dielectric cylinder without "branches". Results are plotted for incidence angles $\theta_{i}=45^{\circ}$ and $-180^{\circ} \leq \phi_{i} \leq 180^{\circ}$. (a) $50 \mathrm{MHz}$, (b) $100 \mathrm{MHz}$, and (c) $300 \mathrm{MHz}$

Figure 9. Currents induced on a tank situated atop Yuma soil with 5\% water content [19]. Results are shown for plane-wave excitation at $500 \mathrm{MHz}$, at $\theta_{i}=45^{\circ}$ and $\phi_{i}=60^{\circ}$, for vertical (V) and horizontal $(\mathrm{H})$ polarization.

Figure 10. Synthetic aperture radar (SAR) imagery for the tank in Fig. 9, for HH polarization, at a depression angle of $\theta_{i}=55^{\circ}$, employing a $60^{\circ}$ SAR aperture and a sensor bandwidth of 50-200 MHz. Results are shown for two target orientations relative to the linear SAR aperture.

Figure 11. Two-dimensional schematic of how the dielectric rough surface is modeled. A finite, flatbottomed dielectric volume is placed in direct contact with an infinite dielectric half space. The electrical properties of the target and bottom half space are identical (representative of soil). The target is designed to constitute a smooth taper from the infinite half space to the rough surface (see Fig. 12).

Figure 12. Three-dimensional rough surface used in example calculations (see Fig. 13). The surface is $7 \times 7$ wavelengths (in the dielectric subsurface).

Figure 13. Bistatic RCS for the surface in Fig. 12, where the soil is characterized by $\epsilon_{r}=5-j 0.2$ and $\sigma=0.01 \mathrm{~S} / \mathrm{m}$, and $f=300 \mathrm{MHz}$. The incidence angles are $\theta_{i}=45^{\circ}$ and $\phi_{i}=0^{\circ}$, and the bistatic RCS is viewed for $0^{\circ} \leq \theta_{s} \leq 90^{\circ}$ and $\phi_{s}=0^{\circ}$. 


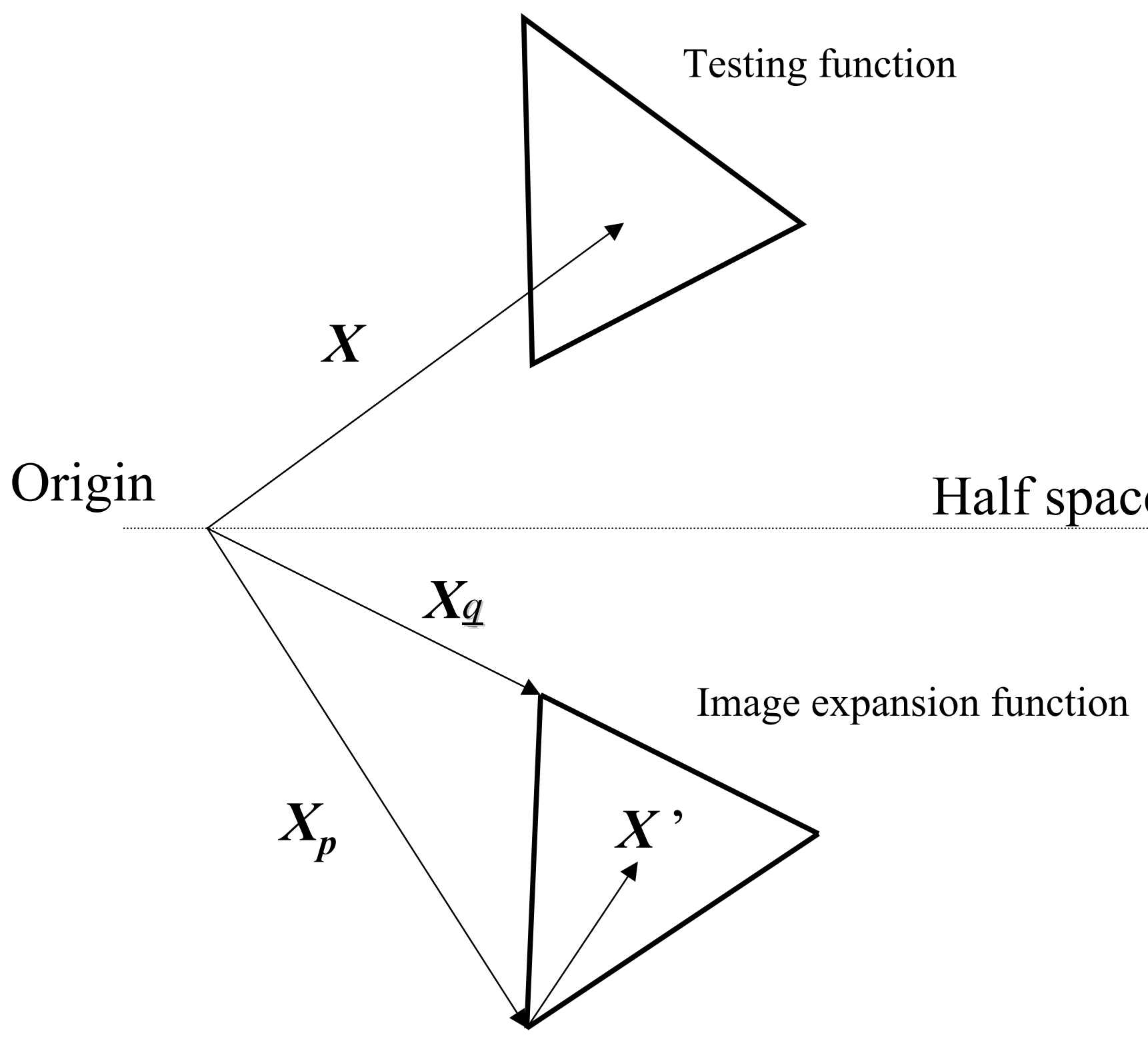

Fig. 1 


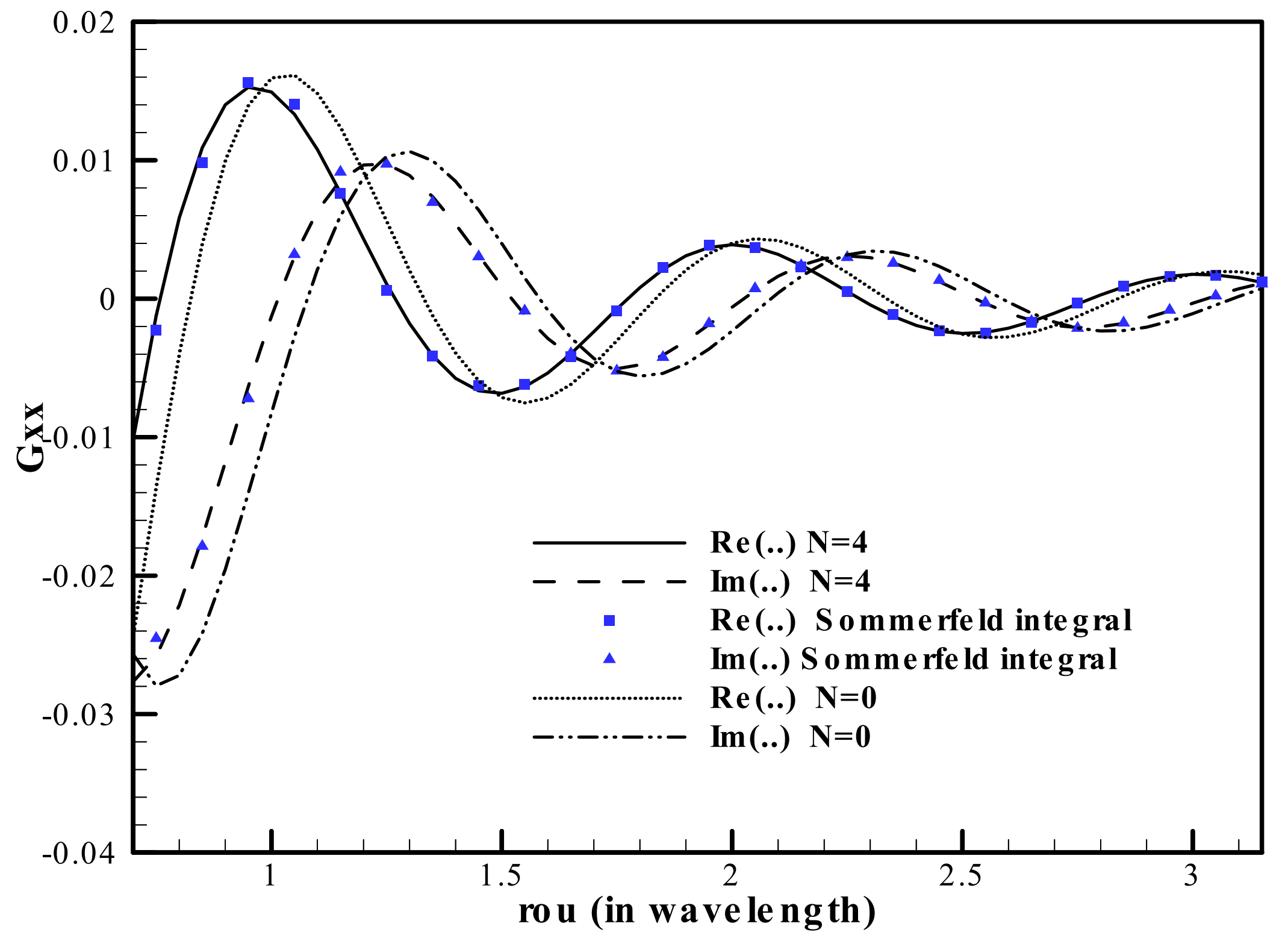

Fig. 2(a) 


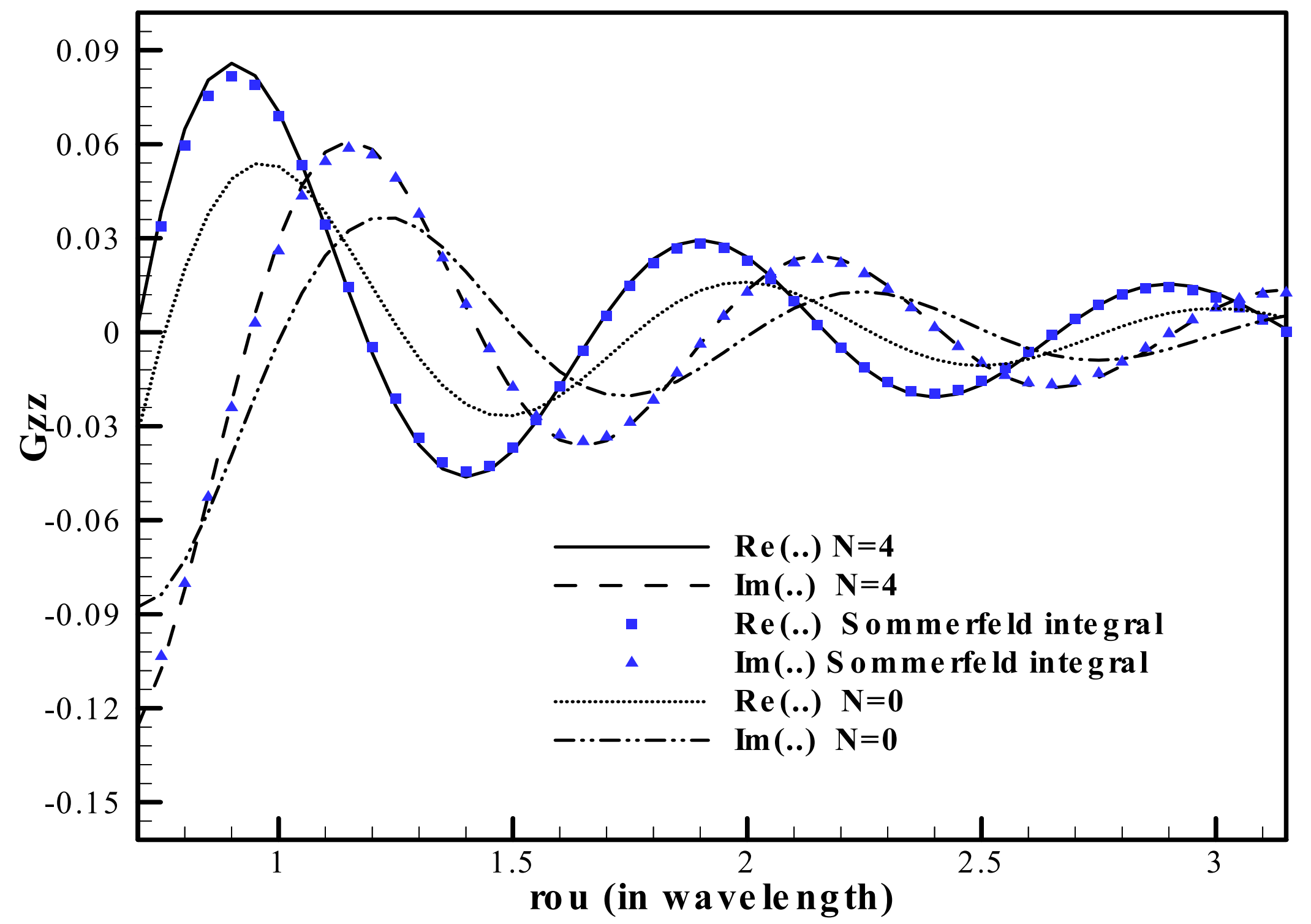

Fig. 2(b) 


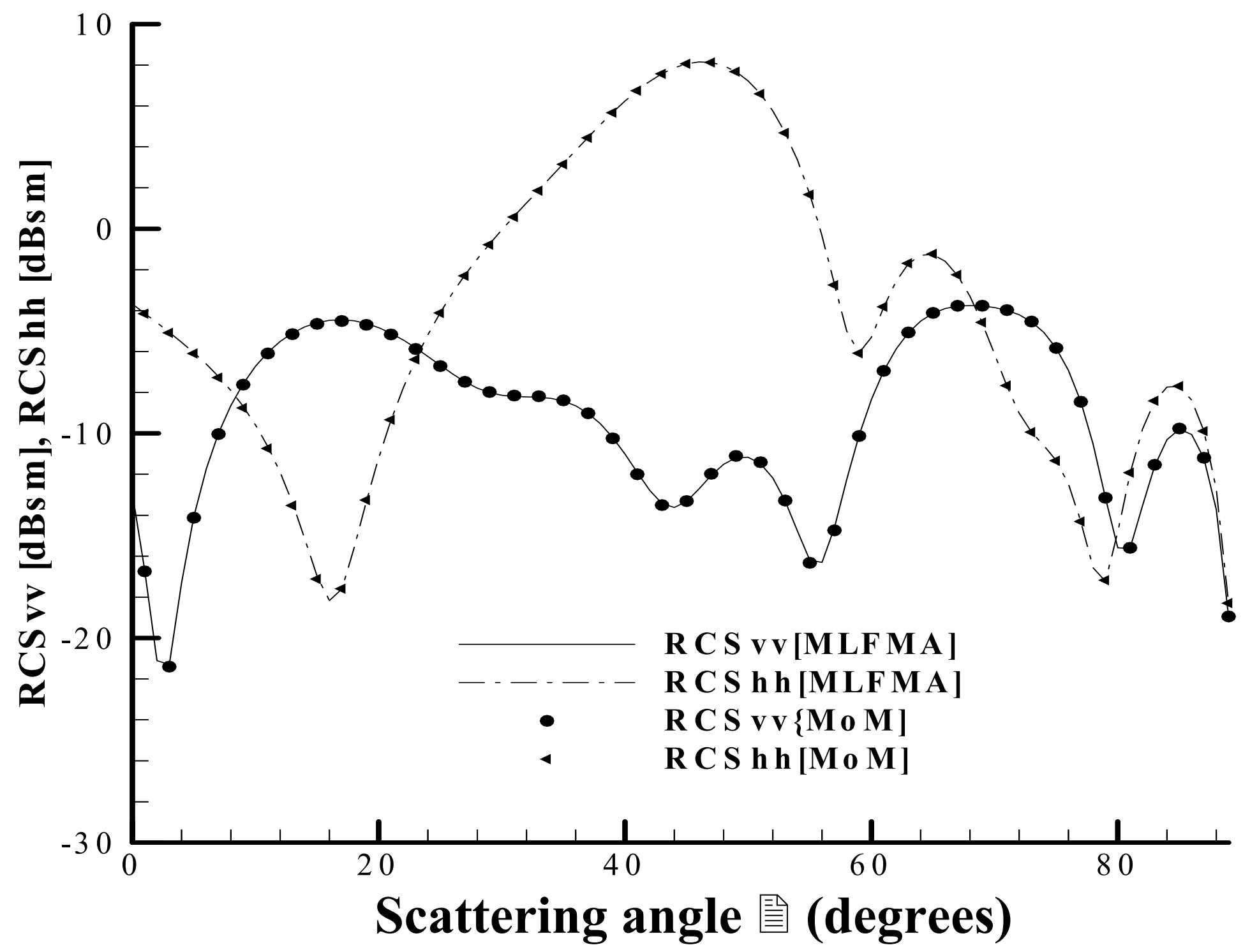

Fig. 3 


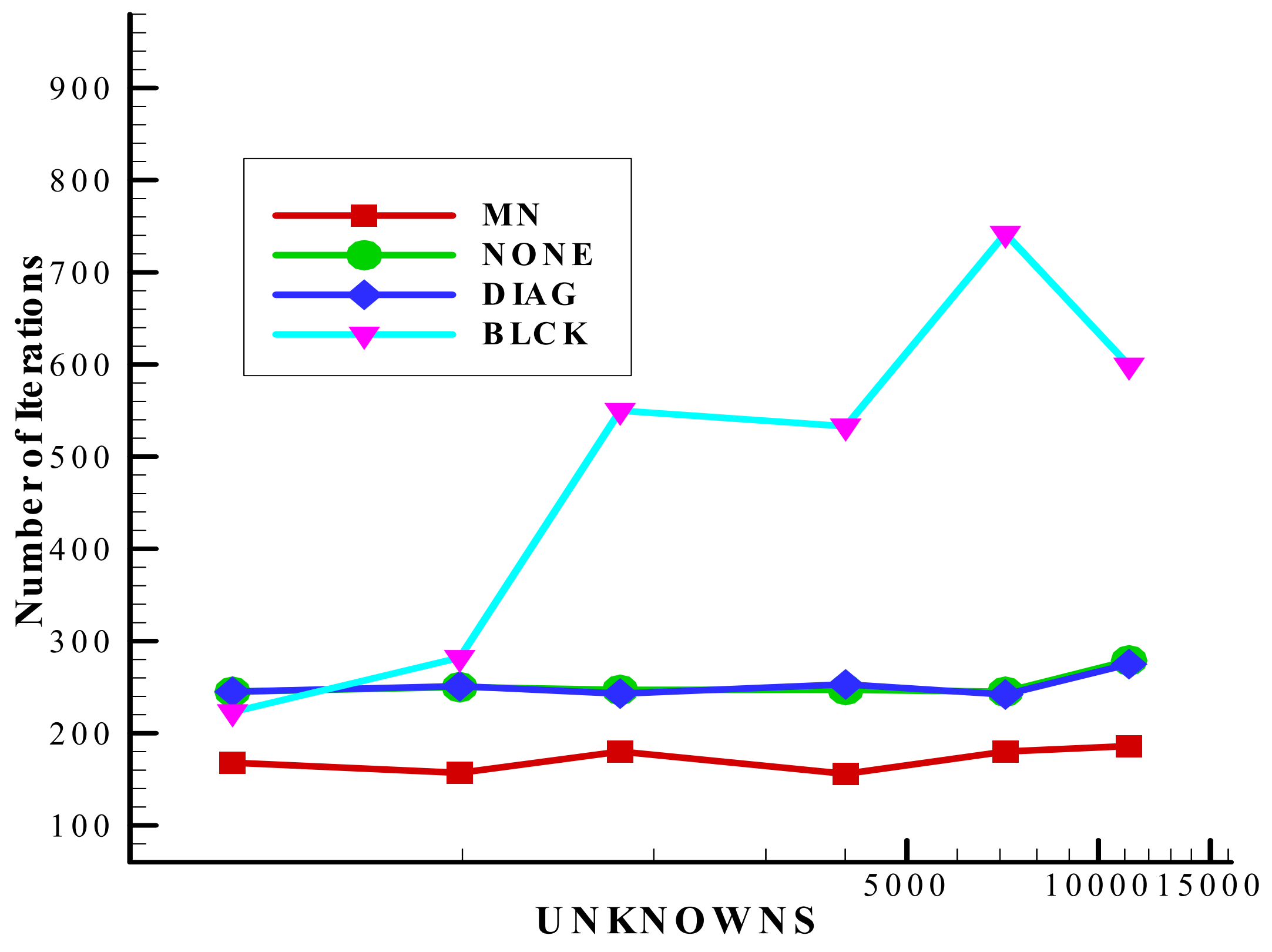

Fig. 4 


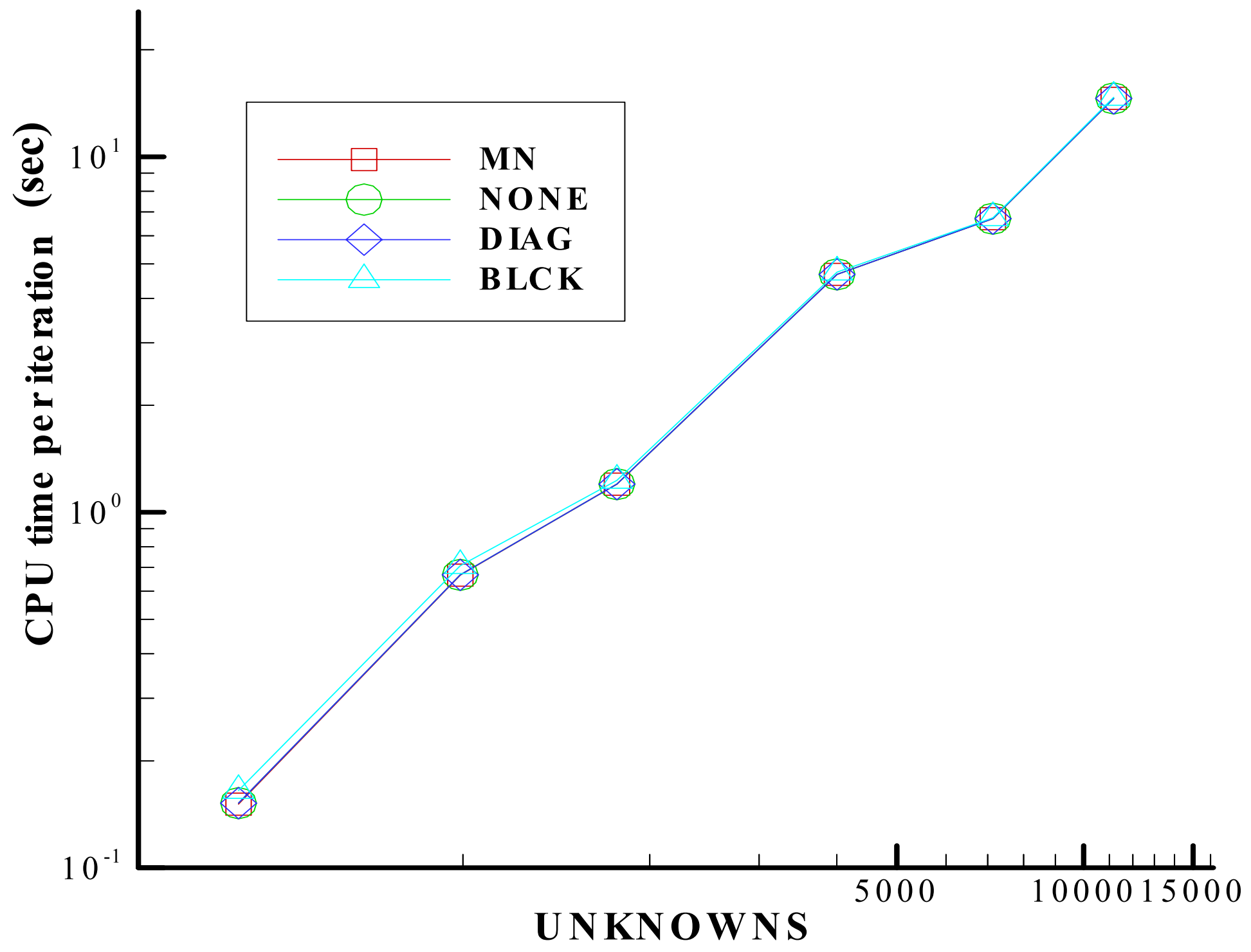

Fig. 5 


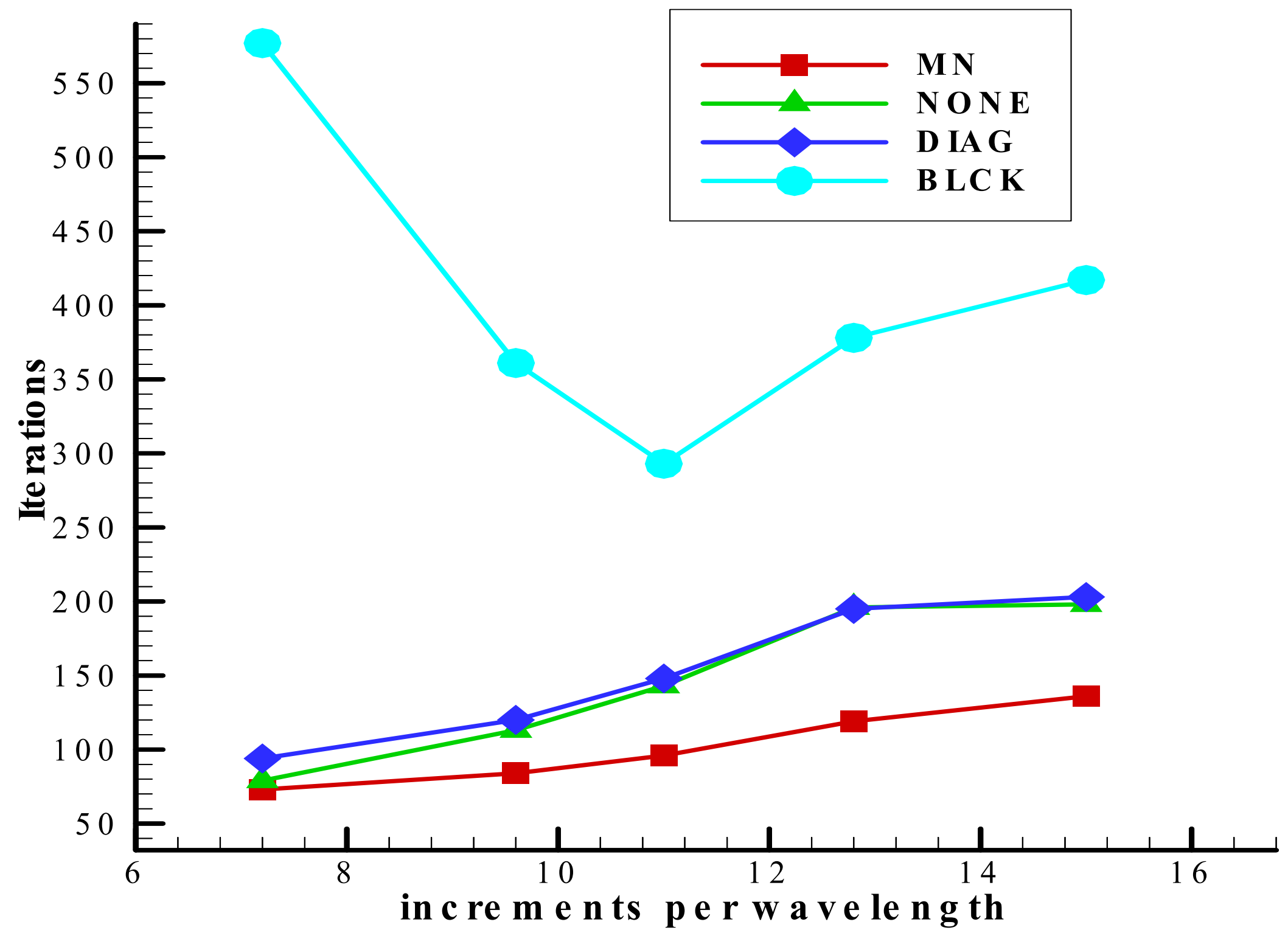

Fig. 6 


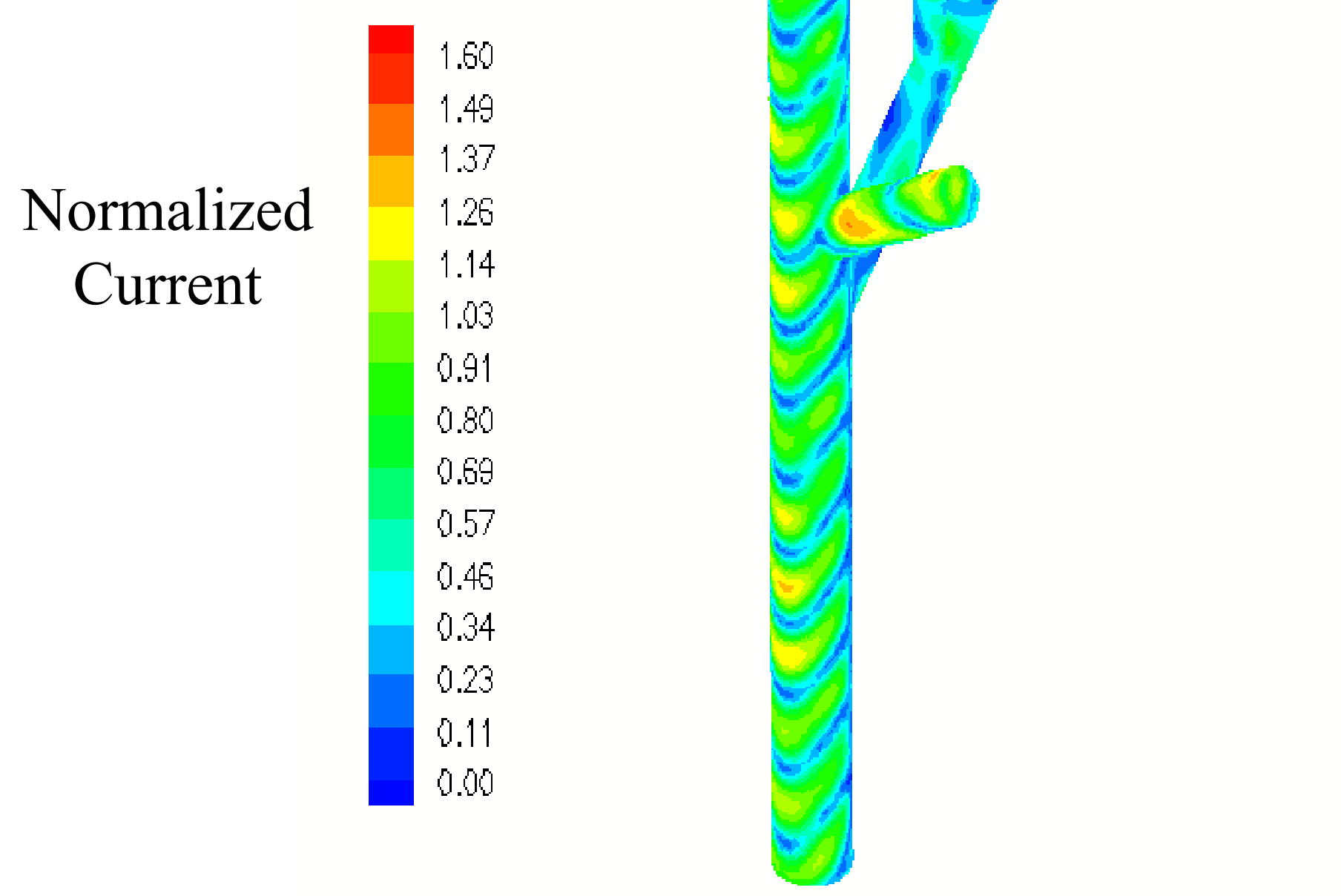

Fig. 7 


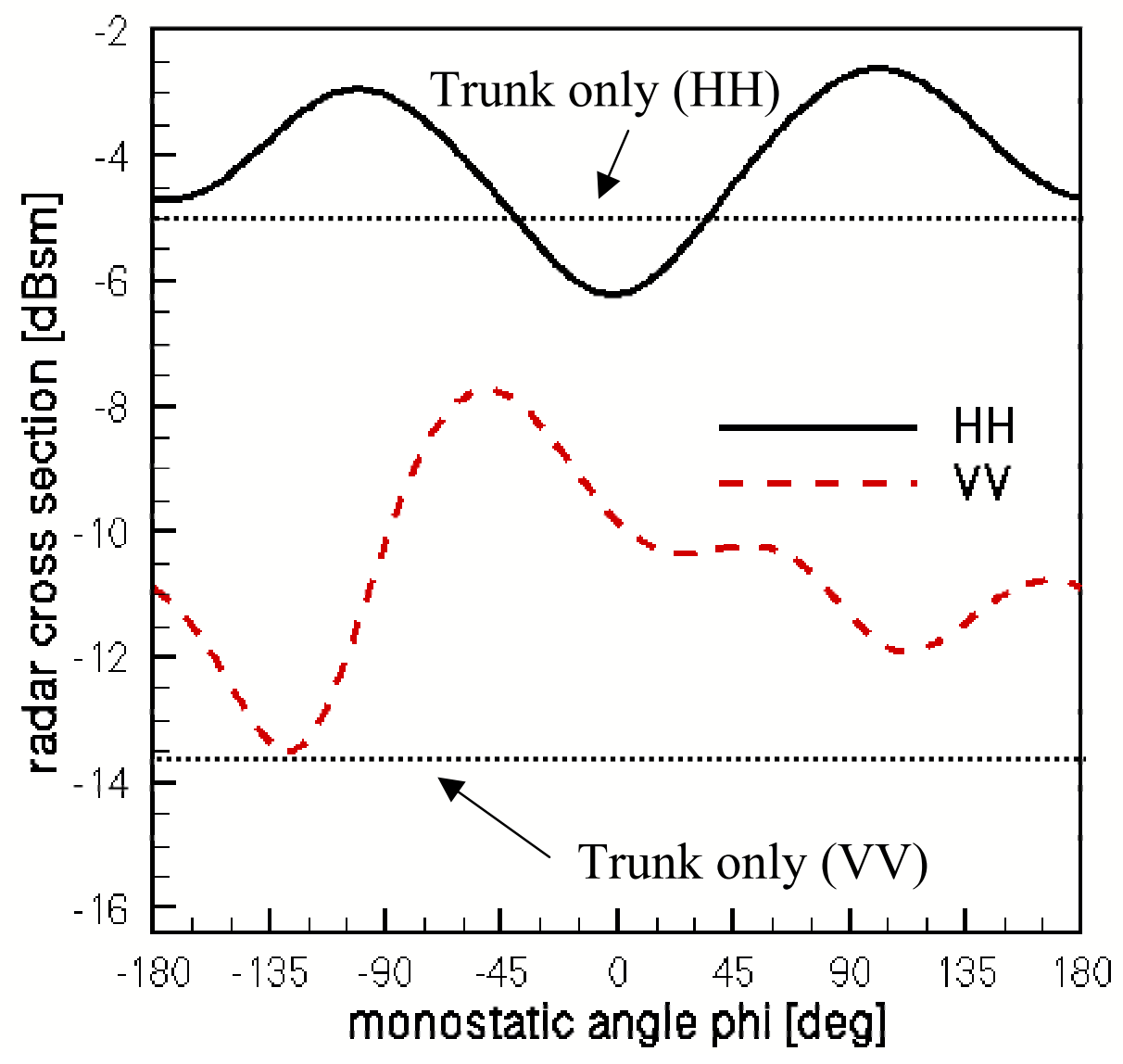

Fig. 8(a) 


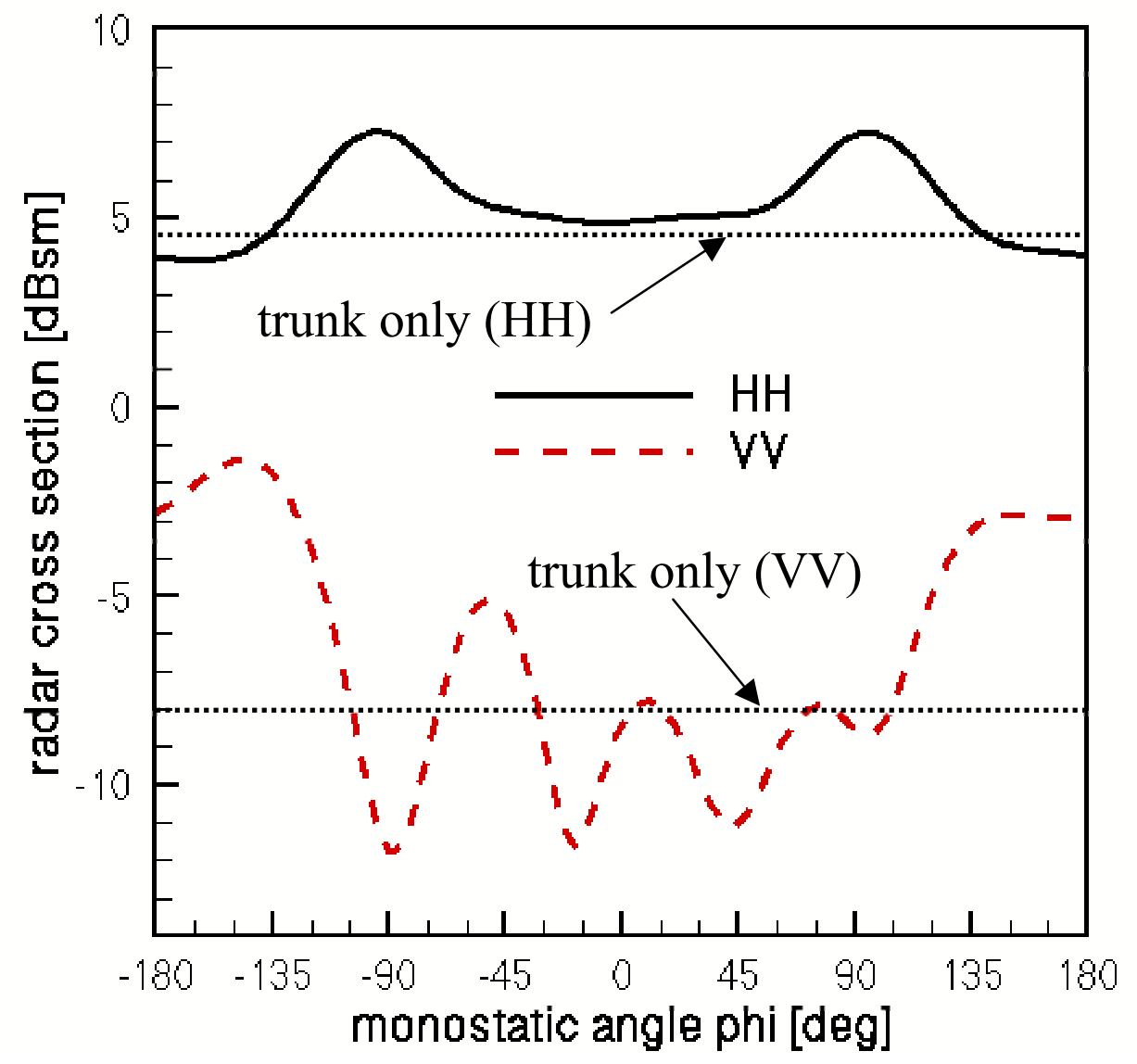

Fig. 8(b) 


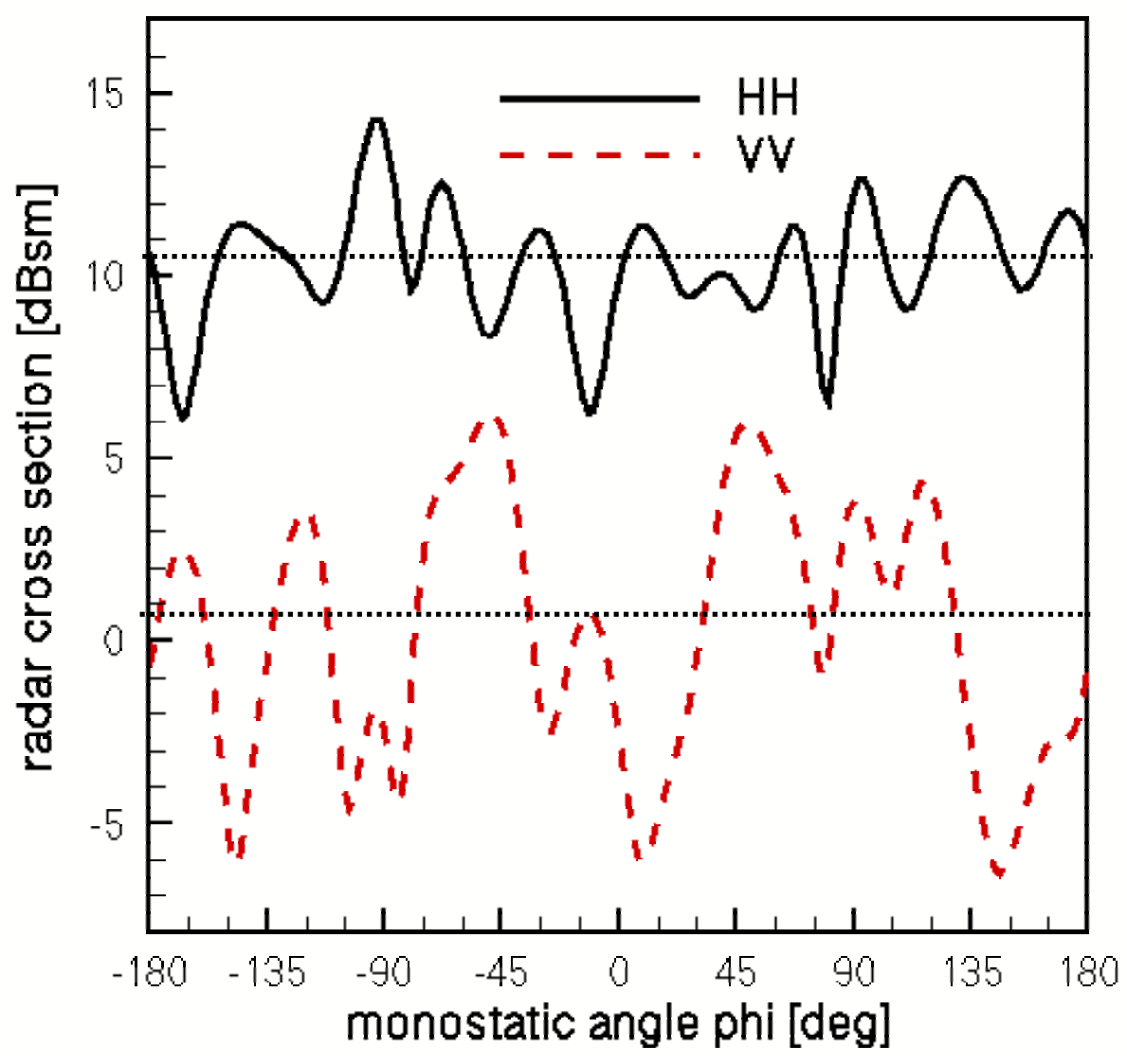

Fig. 8(c) 


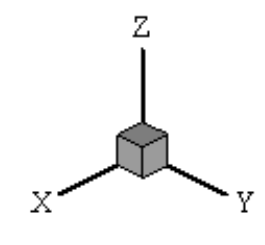

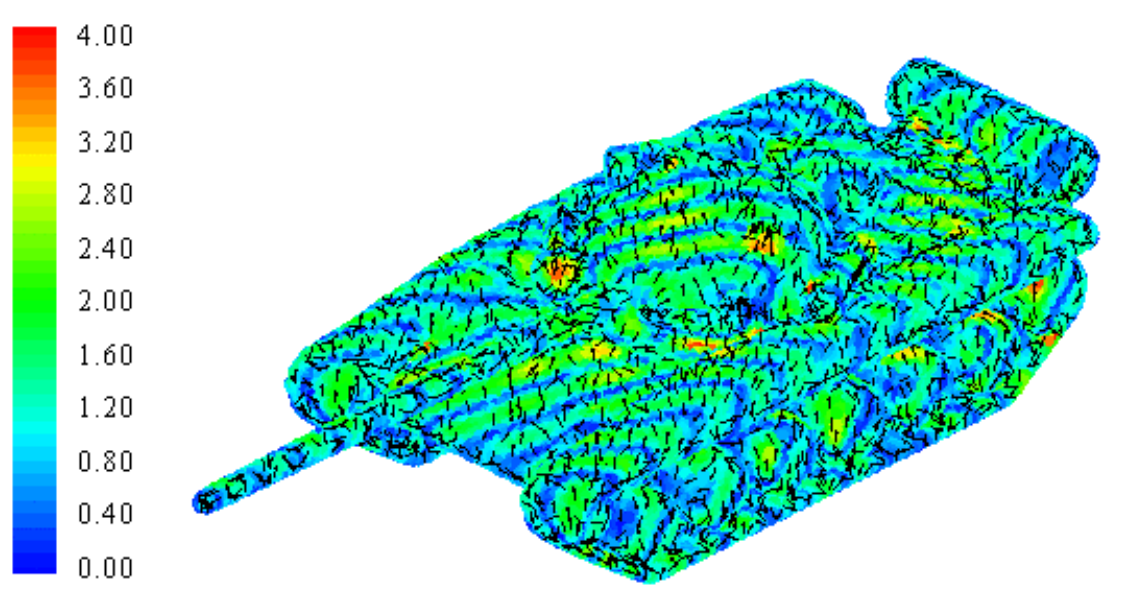

V-pol

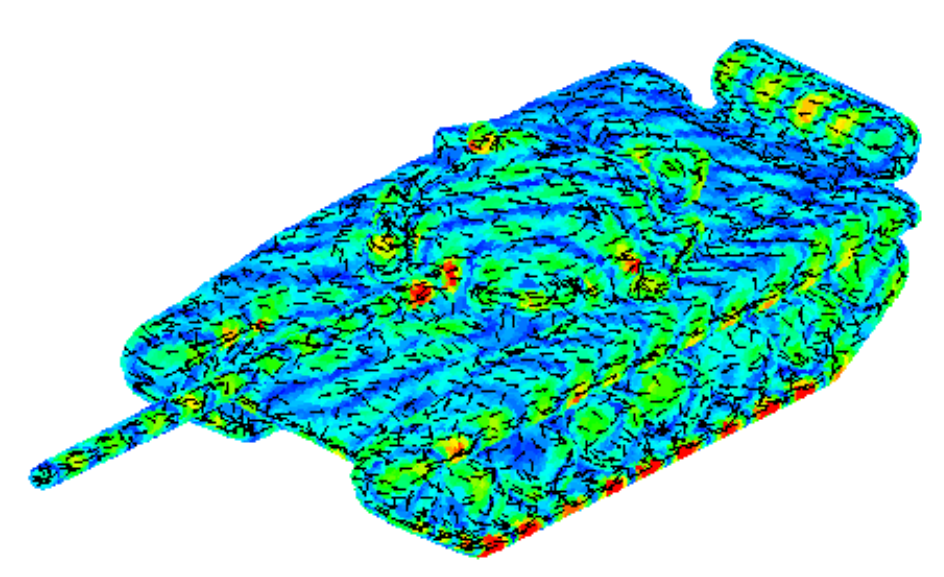

H-pol

Fig. 9 

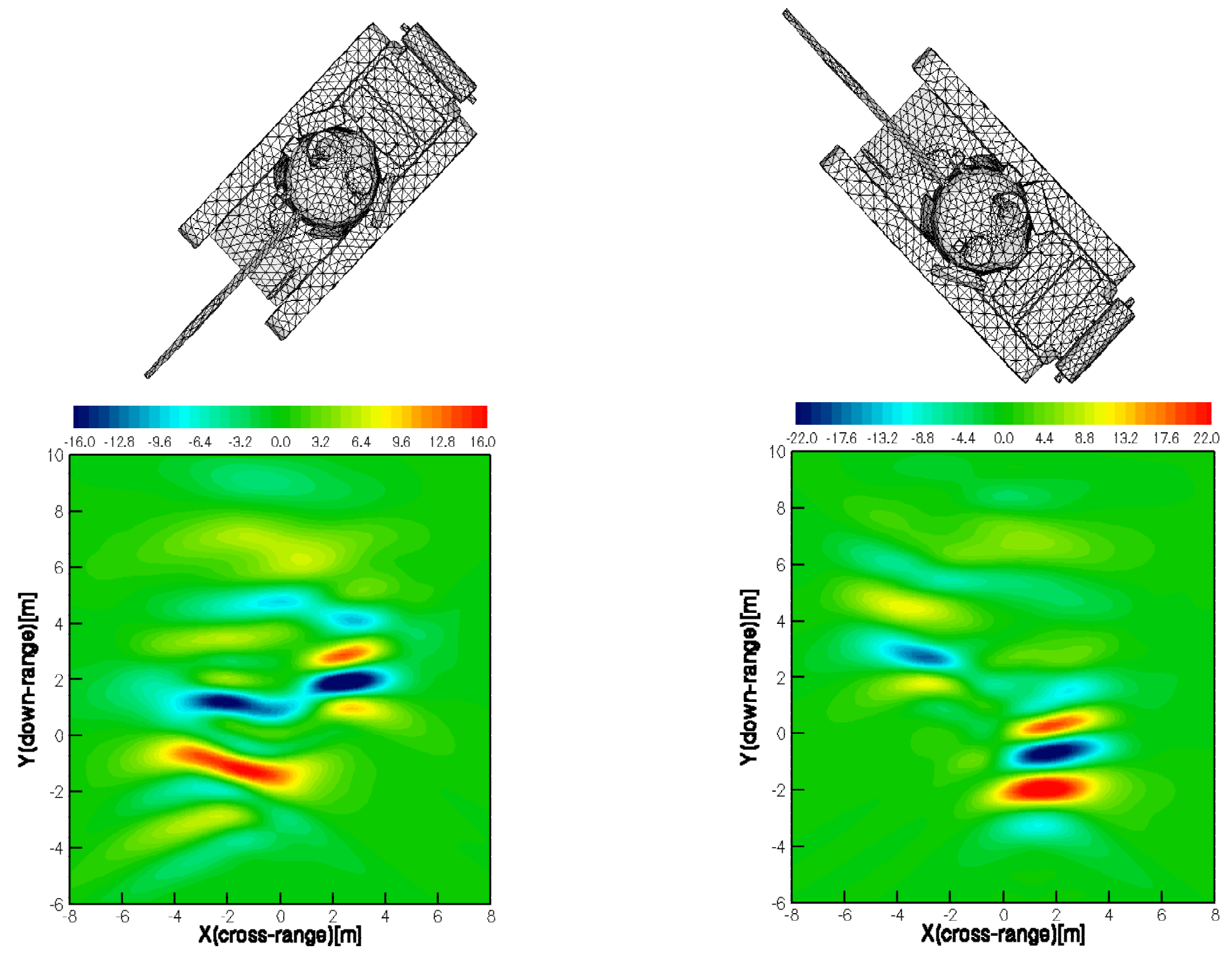

Fig. 10 


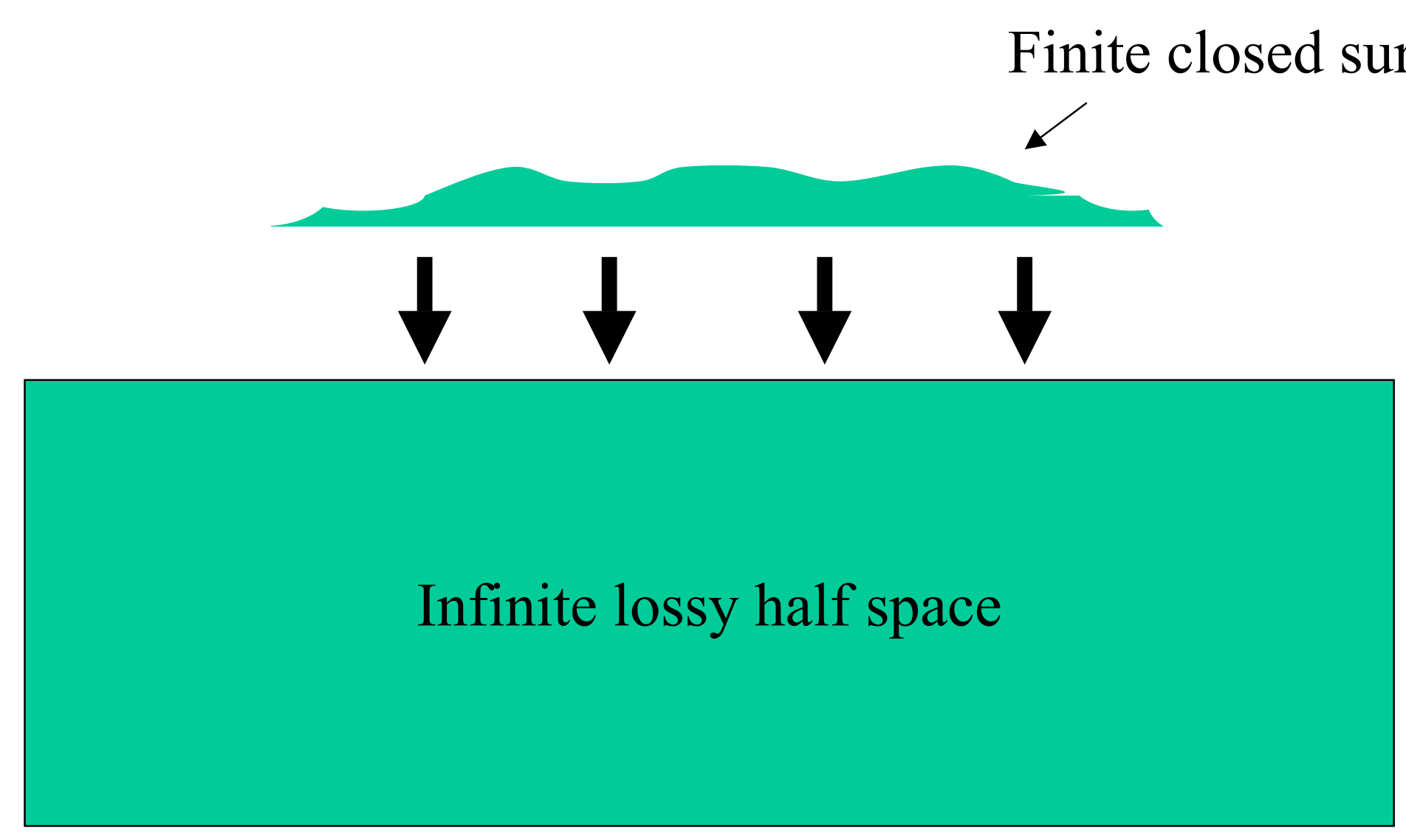

Fig. 11 

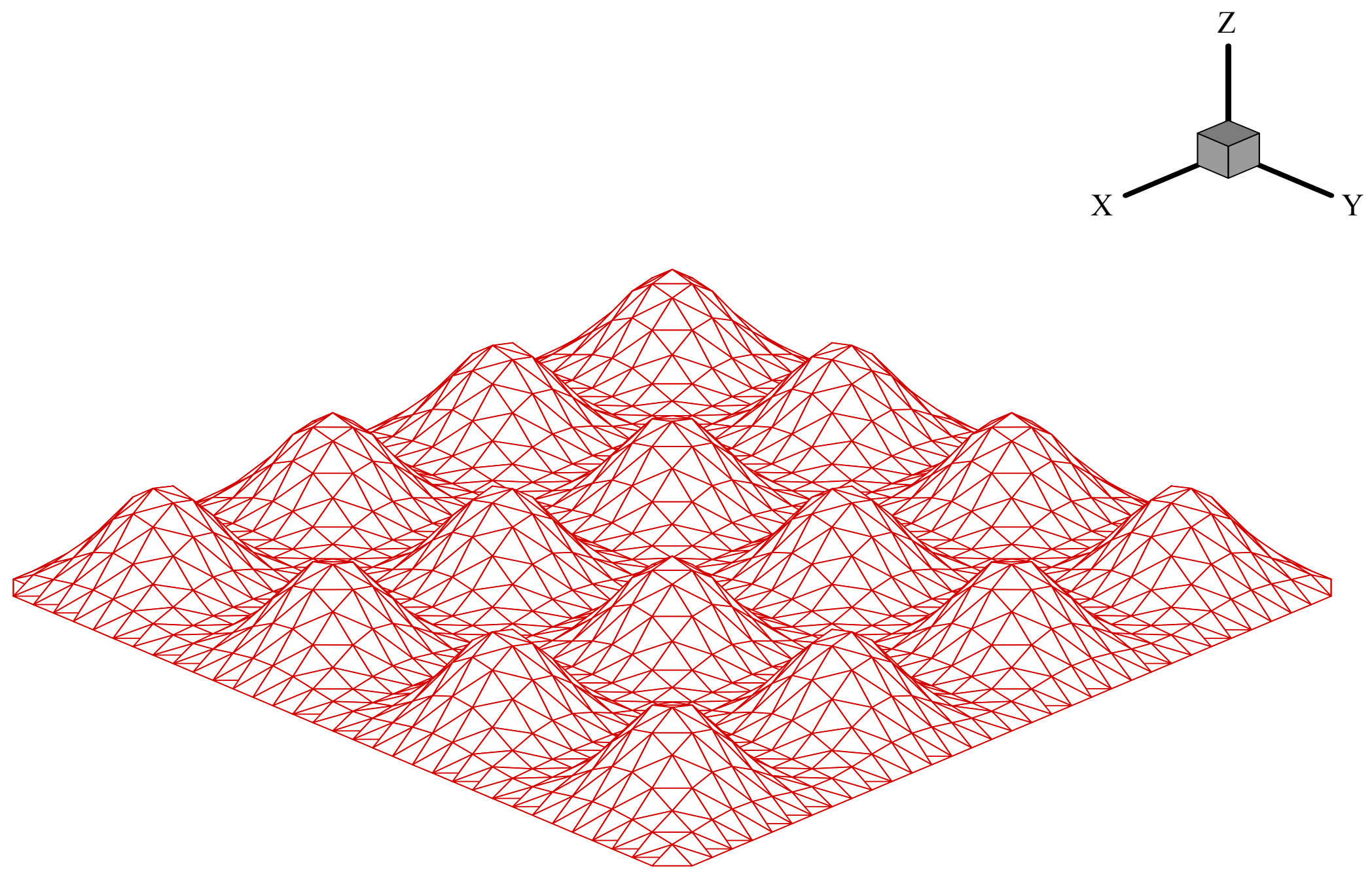

Fig. 12 


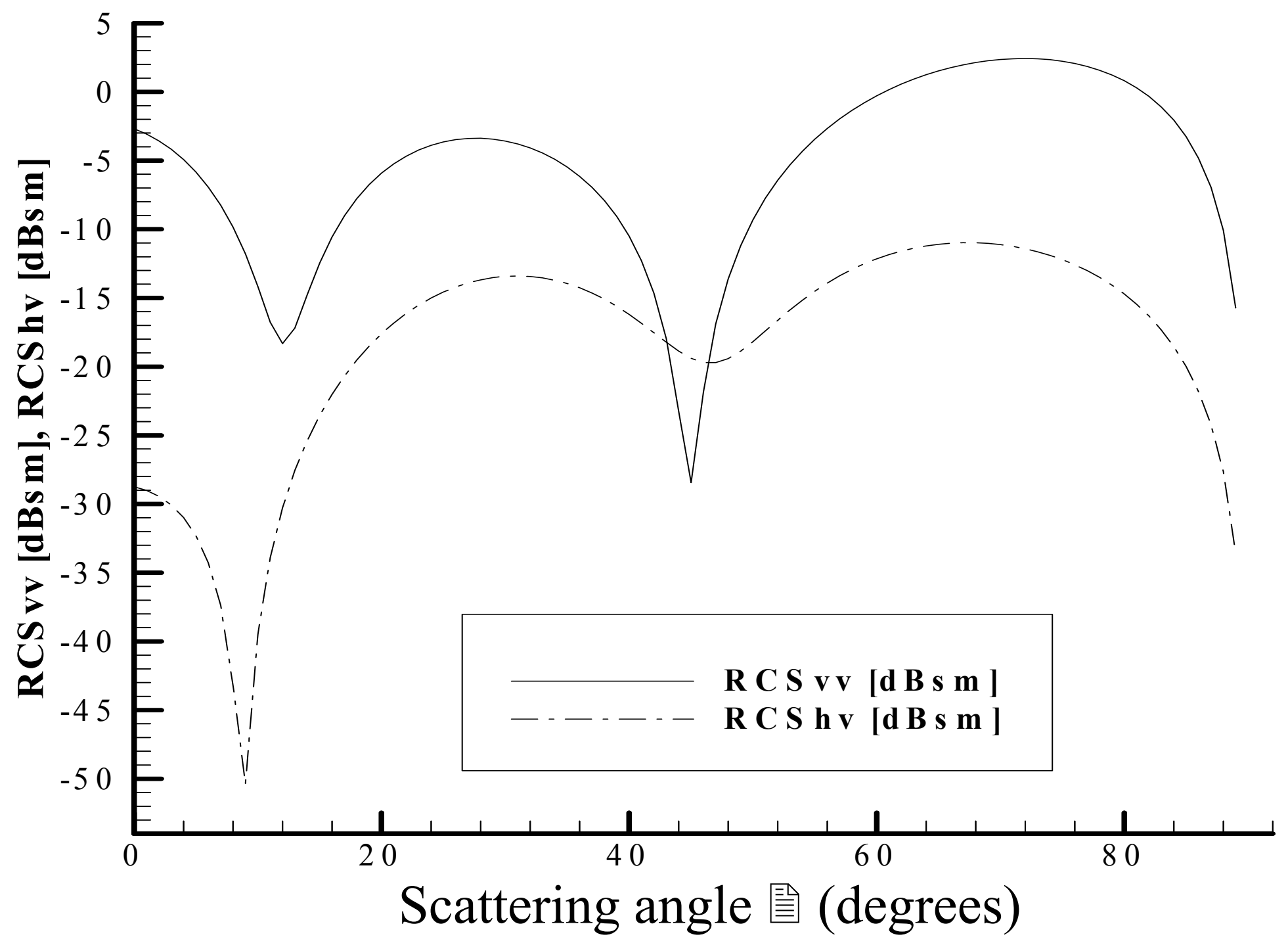

Fig. 13 